\title{
Plants and their active constituents from South, Central, and North America with hypoglycemic activity
}

\author{
José M. Barbosa-Filho*, Tereza H.C. Vasconcelos, Adriana A. Alencar, Leônia M. Batista, \\ Rinalda A.G. Oliveira, Diego N. Guedes, Heloina de S. Falcão, Marcelo D. Moura, Margareth \\ F.F.M. Diniz, João Modesto-Filho
}

\author{
Laboratório de Tecnologia Farmacêutica "Delby Fernandes de Medeiros", Universidade Federal da Paraíba, \\ Caixa Postal 5009, 58051-970, João Pessoa, PB, Brazil
}

\begin{abstract}
There has been marked interest in recent years in the use of plants for the treatment of diabetes. Plants have been found in many countries which have been indicated as having hypoglycemic activity. The present work is an up-to-date review with 178 references of crude plant extracts and chemically defined molecules with hypoglycemic activity from South, Central and North America. The review refers to 224 plants with their families, parts used and type of extract, organism tested and activity. It also includes 40 compounds isolated from those plants. Some aspects of recent research with natural products from plants directed to the treatment of diabetes are discussed.
\end{abstract}

Keywords: Diabetes, hypoglycemic activity, medicinal plants, natural products.

\section{INTRODUCTION}

Diabetes is a disease in which the body does not produce insulin or use it properly. Insulin is a hormone needed to convert sugar, starch and other food into energy needed for daily life. The cause of diabetes continues to be a mystery, althought both genetic and environmental factors such as obesity and lack of exercise appear to play a part.

Worldwide 177 million people suffer from diabetes. This figure is likely to more than double by 2030 (See Table 1). The greater part of the increase is likely to occur in developing countries, which can least afford it.

The annual number of deaths in 2000 caused by diabetes mellitus in Latin America and the Caribbean has been estimated as 339035. This represents a loss of 757096 years of productive life among persons younger than 65 years (Barceló et al 2003). Diabetes is the third leading cause of death in the United States after heart disease and cancer (Acessed from http://www.medicinenet.com/ Diabetes_Mellitus/article.htm in 01/21/2004).

Plants have always been an important source of drugs and many of the currently available drugs have been derived directly or indirectly from them. Ethnobotanical reports indicate about 1200 plants in the world with anti-diabetic potential (Alarcon-Aguilara et al 2002c), of which more than three hundred have been reported in the literature (Perez et al 1984; Almeida et al 1986; Bailey et al 1989; Handa et al 1989; Ivorra et al 1989; Oliveira et al 1989; Rahman et al 1989; Marles et al 1995; Ernest 1997; Pereira 1997; Perez et al 1998b; Volpato et al 2002; Grover et al 2002), referring to a large variety of identified chemical substances (Ivorra et al 1989; Rahman et al 1989; Marles et al 1995; Perez et al 1998b; Lamba et al 2000). The discovery of the widely used hypoglycemic drug, metformin ( $N, N$-dimethylguanylguanidine) came from the traditional approach through the use of Galega officinalis (Grover et al 2002).

In a previous paper this research group has reviewed crude plant extracts and chemically defined molecules with potential antitumor activity for mammary (Moura et al 2001), cervical (Moura et al, 2002) and ovarian neoplasias (Silva et al 2003), as inhibitors of HMG CoA reductase (Gonçalves et al, 2000), central analgesic activity (Almeida et al, 2001), employed in prevention of osteoporosis (Pereira et al, 2002), for the treatment of Parkinson's disease (Morais, 2004) and antileishmanial activity (Rocha et al, 2005).

The present work reviews the literature on plants and plant-derived compounds from South, Central, and North America with hypoglycemic activity. Those plants which are used in the indigenous system of medicine have not been included, except for those whose hypoglycemic activity has been scientifically established.

The search was carred out on Chemical Abstracts, Biological Abstracts, Web of Sciences, LILACS (Latin American and Caribbean literature in Health Sciences) and the data bank of The University of Illinois in Chicago - NAPRALERT (Acronym for Natural Products ALERT), updated to December 2003, using hypoglycemic activity plus anti-diabetic as search terms. The references found in the search were consulted.

The search for data from different sources led 
Table 1. Total of people with diabetes in some countries of South, Central and North America. (Accessed from http://www.int/ncd/dia/databases4.htmin on 01/21/2004)

\begin{tabular}{lrr}
\hline Country & 2000 & 2030 \\
\hline South America & & \\
Argentina & 1426152 & 2457044 \\
Bolivia & 206824 & 554527 \\
Brazil & 4553003 & 11305516 \\
Chile & 494932 & 1047405 \\
Colombia & 883401 & 2410362 \\
Paraguay & 102237 & 324326 \\
Peru & 754087 & 1960957 \\
Central America & & \\
Cuba & 479612 & 875643 \\
Jamaica & 80631 & 197573 \\
Mexico & 2178507 & 6130209 \\
Panama & 59220 & 153308 \\
Trinidad & 60259 & 124780 \\
North America & & \\
Canada & 2006107 & 3542974 \\
USA & 17701942 & 30312264 \\
\hline World & 176525312 & 370023002 \\
\hline
\end{tabular}

to the elaboration of a list of natural products, evaluated specifically for hypoglycemic effect, of several plants and plant-derived compounds, used as anti-diabetic remedies from South, Central and North America (Tables 2-4). It should be noted that most of the references cited are not first hand observations, but compilations copied from other sources. The original references should be consulted for details on the models or mechanism based bioassays used for testing plant extracts and pure compounds for hypoglycemic activity.

\section{Plants and plant-derived hypoglicemic activity}

compounds with

In the Americas many plants are used popularly to control diabetes mellitus. This has caused an increase in the number of experimental and clinical investigations directed toward the validation of the anti-diabetic properties, which have been empirically attributed to these remedies.

In Brazil, around 200 plants are used empirically to control diabetes mellitus. Of these, fifty two have been experimentally studied and hypoglycaemic activity detected in most of them (See Table 2). Bauhinia forficata known popularly as "pata-de-vaca" (cows hoof) is the most studied species. Some studies confirm the activity and others do not. This controversy may be related to the model employed in the experiments. More recently, Pepato et al. (2002) analysed the effects of a leaf decoction as a driking-water substitute for about 1 month on streptozotocin-diabetes (STZ-diabetes) in male Wistar rats. The STZ-diabetic rats treated with the decoction showed a significant reduction in serum and urinary glucose as compared with the STZ-diabetic control, no difference being seen between decoction-treated and untreated non-diabetic rats.

In Venezuela, the aqueous extract of $B$. megalandra has been used for diabetes mellitus. It was shown to inhibit intestinal glucose absorption in a concentration-dependent way and additive to phlorizine (Gonzalez-Mujica et al., 2003). In addition, the Chilean species $B$. candicans also used for diabetes, presented a significant decrease of glycaemia in alloxan diabetic rats (Lemus et al., 1999).

Phyllanthus sellowianus is a plant used in folk medicine in Argentina as a hypoglycemic and diuretic agent. The aqueous and butanolic extract of this plant, administered at a dose of $200 \mathrm{mg} / \mathrm{kg}$ p.o., caused a significant reduction in blood glucose concentration after 6 and $9 \mathrm{~h}$ in mice, similar to that observed with glibenclamide $(10 \mathrm{mg} / \mathrm{kg})$ which was used as a reference, while the dichloromethane extract of the same plant was ineffective (Hnatyszyn et al., 2002).

Aproximately 150 plants are used in traditional folk medicine in the treatment of diabetes in Mexico (Alarcon-Aguilar et al., 1998). However, only a small number of them have been studied scientifically. The plants most extensively studied are "nopal" Opuntia streptacantha, "tronadora" Tecoma stans, "Guarumbo" Cecropia obtusifolia and "Matarique" Psacalium decompositum, (see Table 3). The aqueous extract of the latter species significantly reduced blood glucose in a dosedependent manner in normal mice after intraperitoneal administration $(P<0.05)$ (Alarcon-Aguilar et al., 2000).

A menu which includes common culinary herbs and spices with hypoglycemic activity for the control 
<smiles>CC1(C)CCC2(C(=O)O)CC[C@H]3C(=CCC4C5(C)CCC(O)C(C)(C)C5CCC43C)C2C1</smiles><smiles>C[C@H]1CCC[C@@H]2C=CC(=O)CO[C@@H](c3ccoc3)C[C@H]12</smiles><smiles>COc1ccc(-c2oc3cc(O)c(OC)c(O)c3c(=O)c2OC)cc1O</smiles>

5

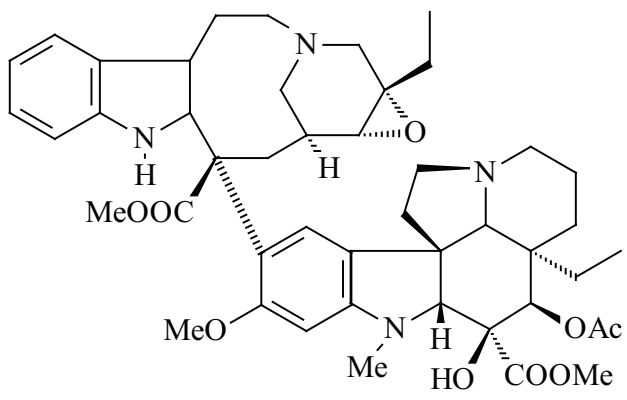

7<smiles>C=C[C@](C)(CCC=C(C)C)CCc1ccc(O)cc1</smiles><smiles>CC1(C)CCC2(C(=O)O)CCC3C(=CCC4C5(C)CC(O)C(O)C(C)(CO)C5=CCC34C)C2C1</smiles><smiles>C=C1C[C@]23CCC4(C(=O)O)[C@@H](CCCC4(C)C)[C@H]2CCC1(O)C3</smiles>

4<smiles>O=c1cc(-c2ccc(O)c(O)c2)oc2cc(O)c(C3OC(CO)C(O)C(O)C3O)c(O)c12</smiles>

6

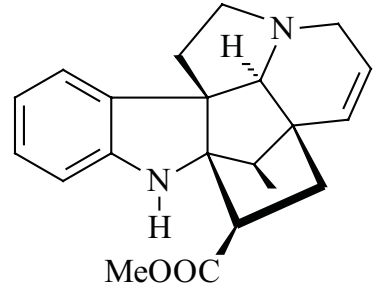

8

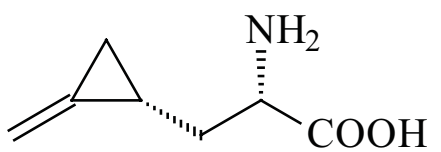

10

Figure 1. Representative examples of compounds with hypoglycemic activity. 
and prevention of diabetes mellitus was utilized by Broadhurst et al. (2000). To evaluate the possible effects on insulin function, 49 herb, spice, and medicinal plant extracts were tested in the insulin-dependent utilization of glucose using the rat epididymal adipocyte assay. "Cinnamon" Cinnamomum cassia was the most bioactive product followed by witch hazel Hamamelis virginiana, green and black teas Camellia sinensis, allspice Pimenta officinalis, bay leaves Laurus nobilis, nutmeg Myristica fragans, and cloves Syzigium aromaticum (see Table 4).

A survey of the literature has shown that a large variety of compounds obtained from several plants of South, Central and North America were found to possess hypoglycemic action. For instance, the triterpenes oleanolic acid (1) and bassic acid (2) from Bouvardia terniflora (Perez et al., 1998) and Bumelia sartorum (Naik et al., 1991) respectively lowered blood sugar in test animals. Similarly the diterpenes trans-dehydrocrotonin (3) from Croton cajucara (Farias et al., 1997) and steviol (4) from Stevia rebaudiana (Ishii; Bracht, 1985) exhibited similar activity. Certain flavonoids eg. 5,7,3trihydroxy-3,6-4'-trimethoxyflavone (5) from Brickellia veronicaefolia (Perez et al., 2000a) and the glycoside isoorientin (6) from Cecropia obtusifolia (AndradeCetto et al., 2001) also showed hypoglycaemic effects. A number of alkaloids isolated from Vinca rosea (Catharanthus rosea) with antitumor activity (Svoboda et al., 1964) were submitted for assay for hypoglycemic effects. The results indicated that catharantine, leurosine (7), lochnerine, tetrahydroalstonine, vindoline and vindolinine (8) produce varying degrees of blood-sugar reduction. For Otholobium pubescens this property was attributed to a phenolic compound bakuchiol (9). The amino acid hypoglycine A (10) isolated from Blighia sapida was particularly effective against diabetes (Kean, 1975; Mills et al., 1987) (Figure 1). The great variety of chemical classes indicate that a variety of mechanisms of action are involved in reduction of the glucose level in blood.

The information recorded in Tables 2-4, has been assembled by continent (South, Central and North America), with the name of the country, plant in alphabetical order, scientific name, family, part used, organism tested, activity and reference. This study has enumerated 224 plants and 40 compounds for which hypoglycemic activity has been reported, as a result of pharmacological studies carried out in various research centers in Argentina, Brazil, Canada, Chile Colombia, Cuba, Jamaica, Mexico, Panama, Paraguay, Peru, Puerto Rico Trinidad and the USA. The ten principal families in which such activity has been reported are Fabaceae (25), Asteraceae (25), Myrtaceae (11), Labiatae (10), Cucurbitaceae (8), Solanaceae (7), Anacardiaceae (6), Euphorbiaceae (6), Rubiaceae (6), and Liliaceae (5).
This literature review adds more data to that previously published, since there are many plants in South, Central and North America, which present hypoglycemic effects.

The number of plants described in the literature as having hypoglycemic activity is more or less the same in the three continents. Among the 224 plants studied $73(33 \%)$ are found in South America, 77 (34\%) in Central America and 74 (33\%) in North America. The countries in each continent with the largest contribution are: Brazil with 52 (23\%) of the plants studied, Mexico with $54(24 \%)$ and the USA with $70(31 \%)$. None of the plants with hypoglycemic activity was found in all three continents. The following species stood out for the number of citations reported in the scientific literature in each continent: Bauhinia forficata with 8 citations (Brazil), Opuntia streptacantha with 5 citations (Mexico) and, Avena sativa (USA) with 3 citations.

Of an estimated 250.000 higher plants, less than $1 \%$ have been screened pharmacologycally and very few in regard to diabetes mellitus. Therefore, it is prudent to look for options in herbal medicine for diabetes mainly in developing countries because it is a pathological condition associated with high morbidity, mortality and economic impact. None of the plants used in traditional medicine, should be used until safety studies have been completed.

\section{ACKNOWLEDGEMENTS}

The authors wish to express their sincere thanks to the College of Pharmacy of the University of Illinois at Chicago, Chicago, Illinois 60612-7231, U.S.A., for helping with the computer aided NAPRALERT and CNPq/FAPESQ-PB/Brazil for financial support.

\section{CONCLUSION}




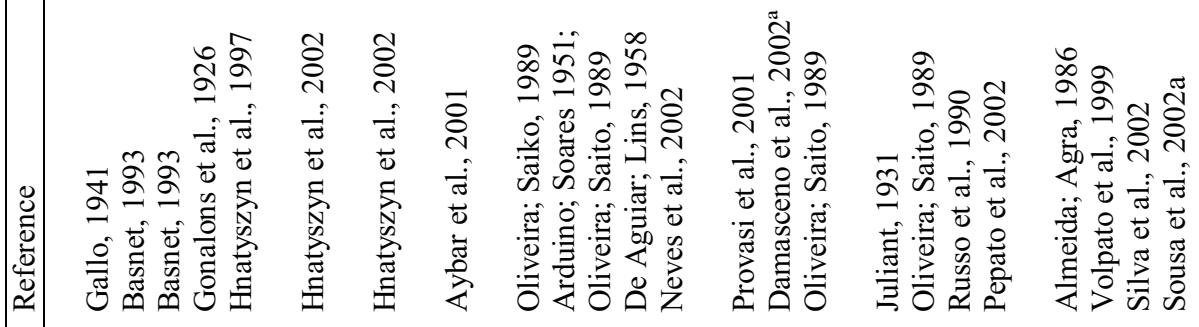

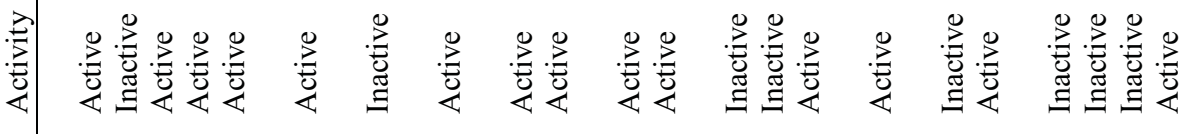

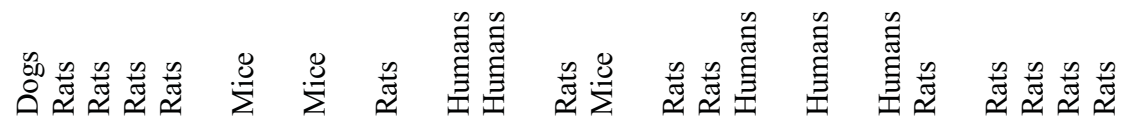

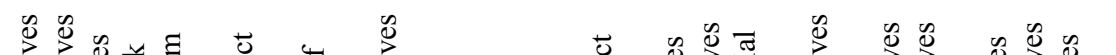

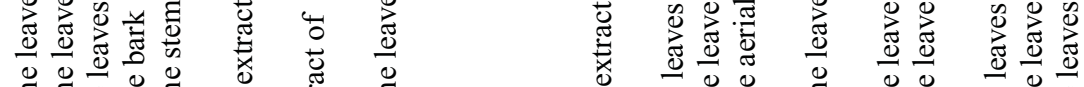
gᄋ

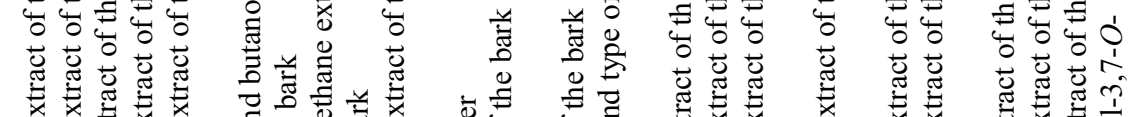

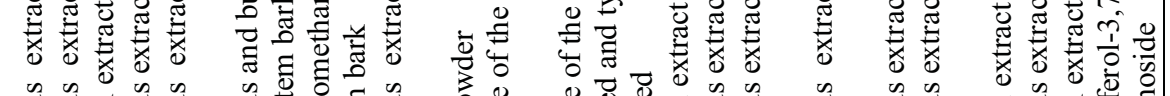

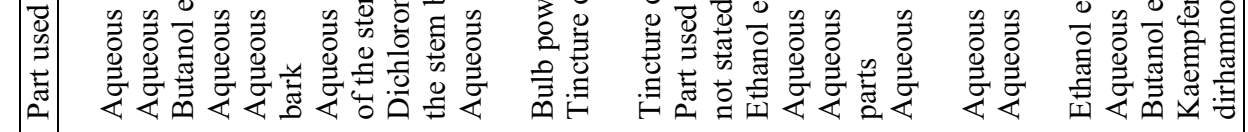

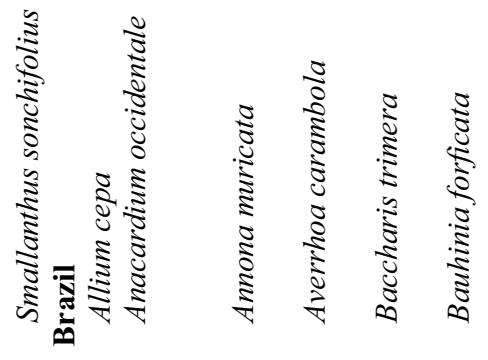


œ ڤ ڤ̆

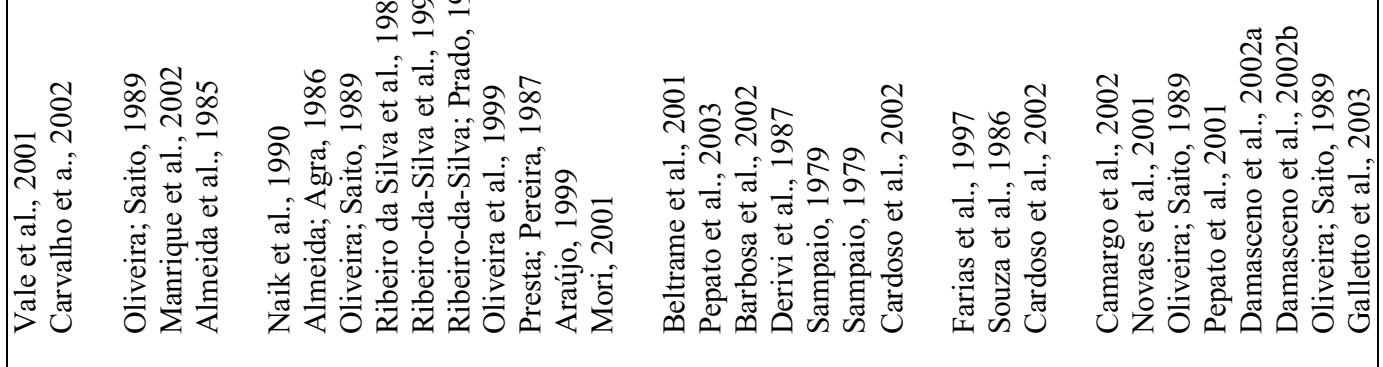

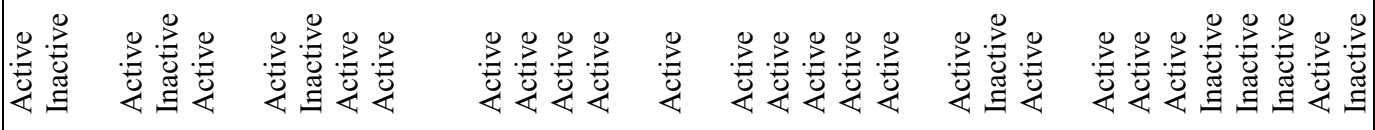

.

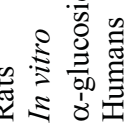

芯芯

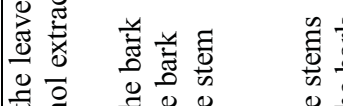

吾

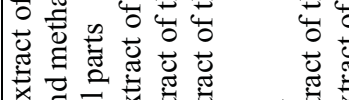

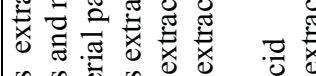

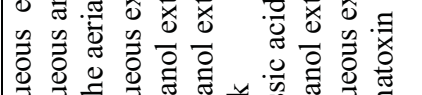

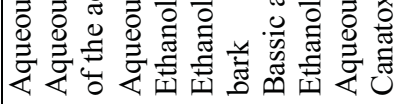

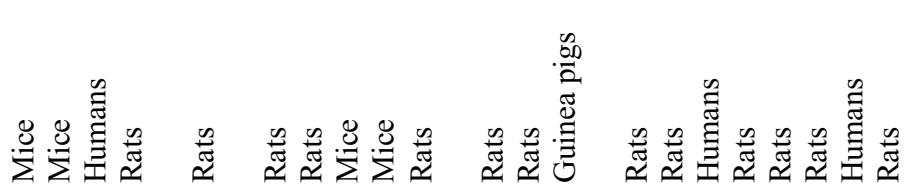

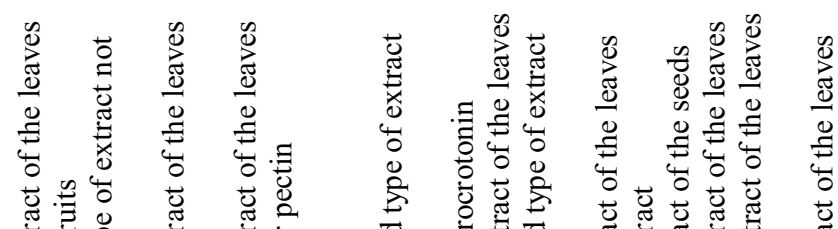

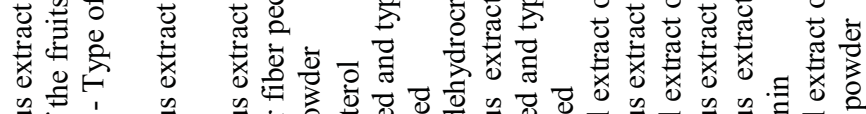

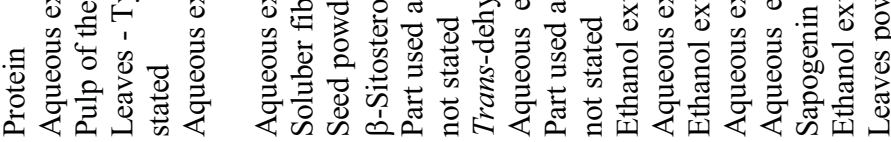

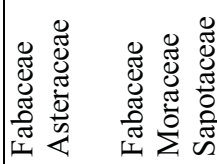

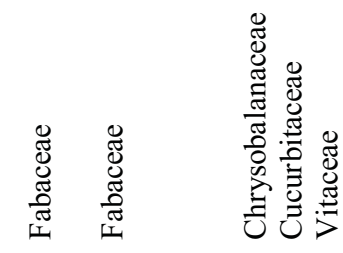

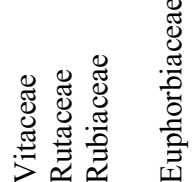
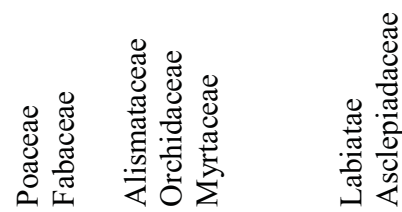

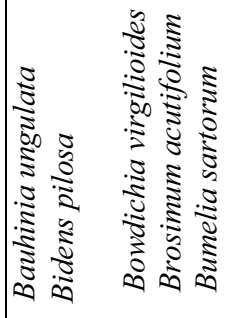
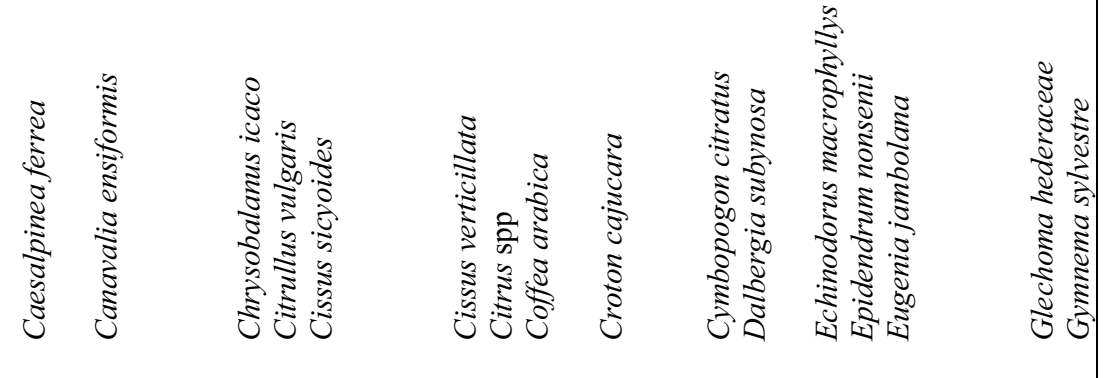


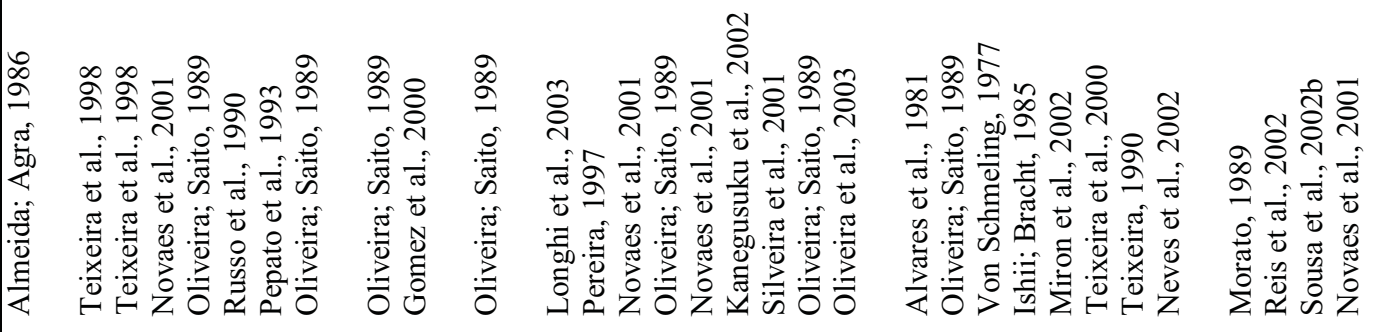

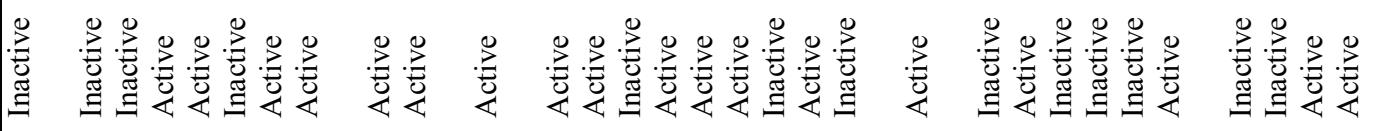

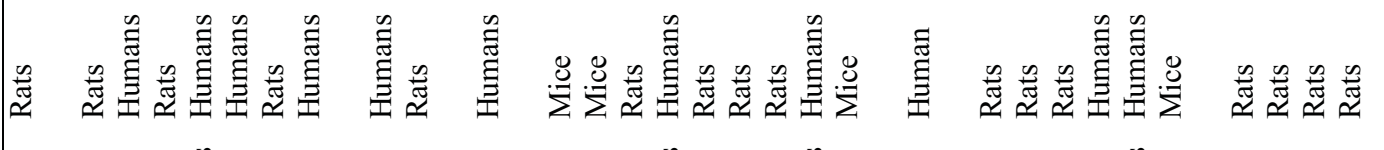

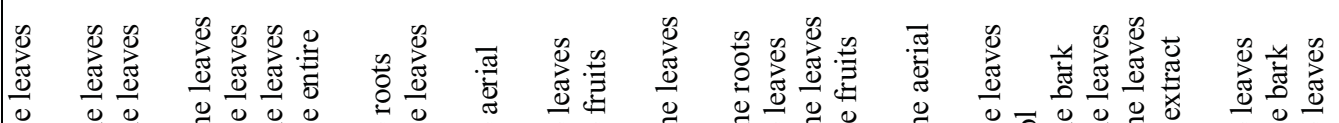

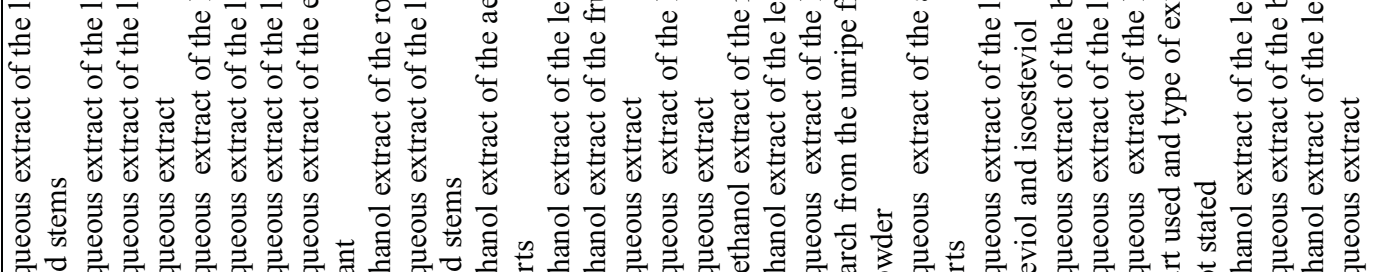

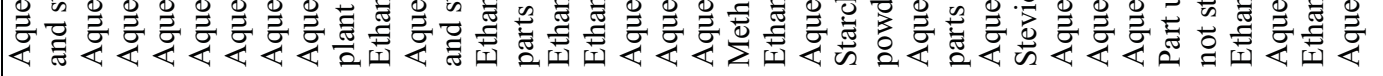

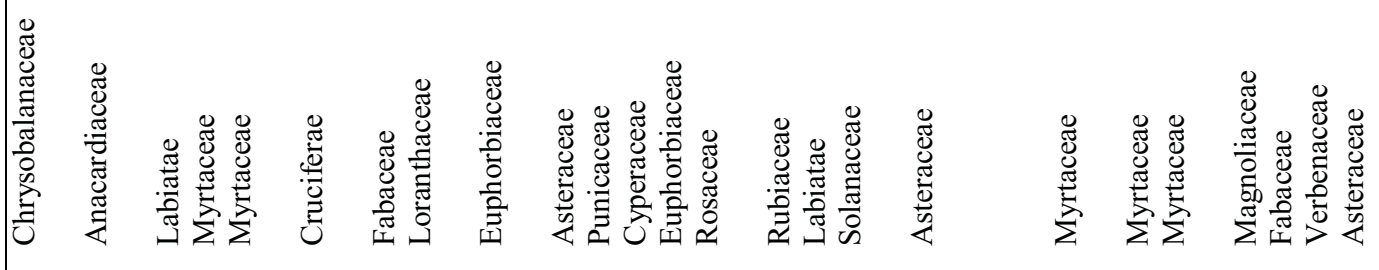

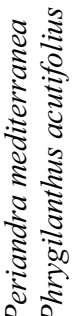
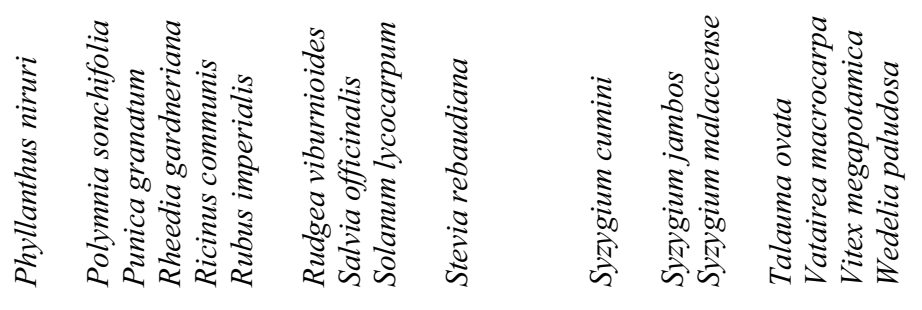


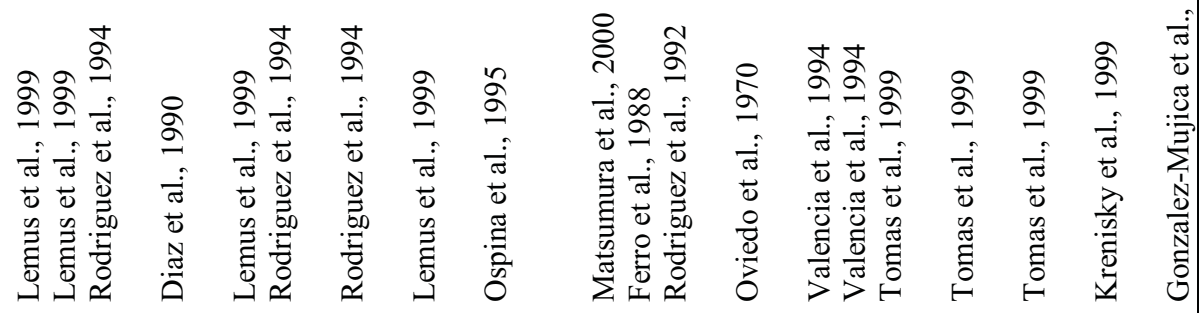

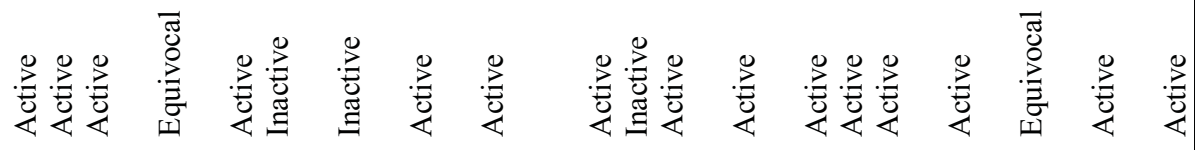

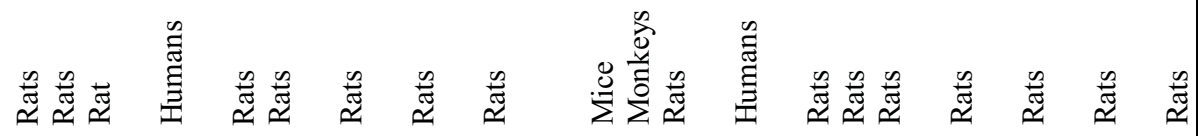

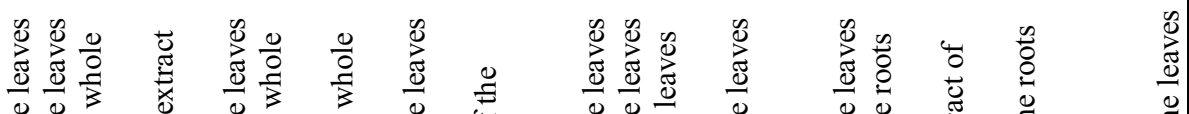

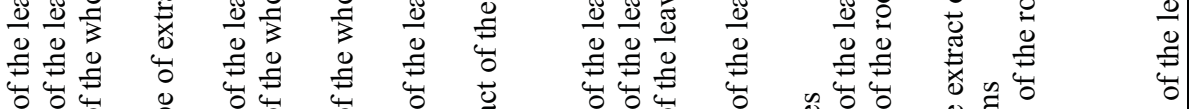

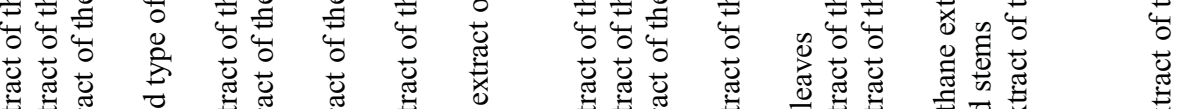

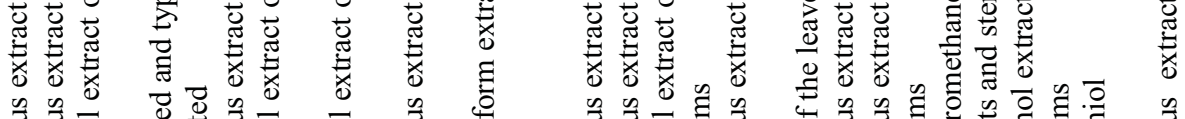

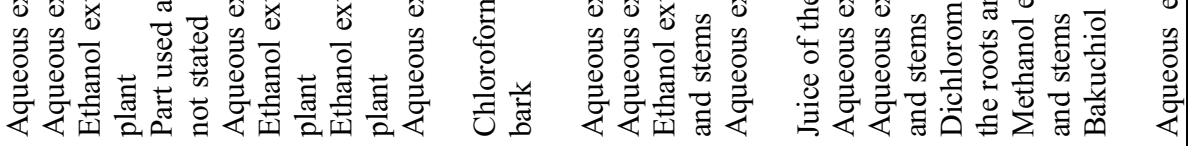

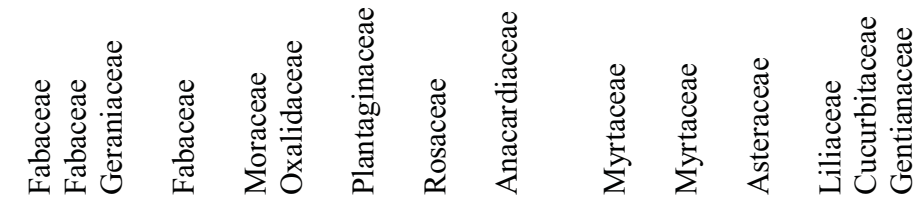

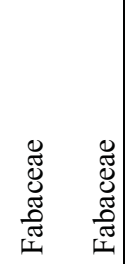

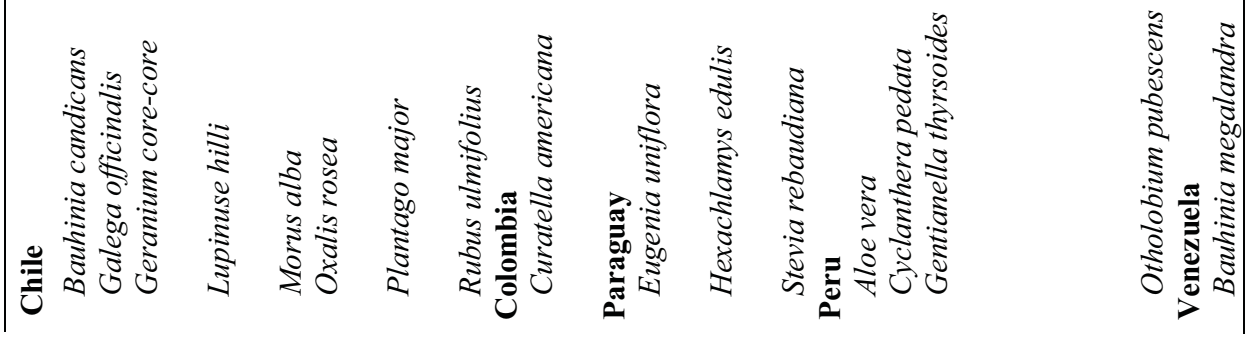




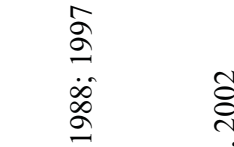

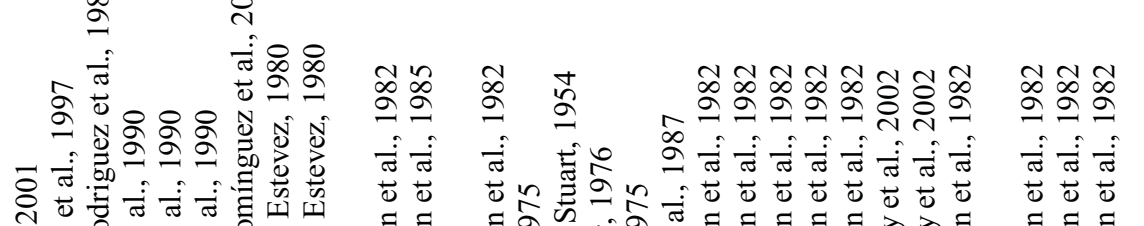

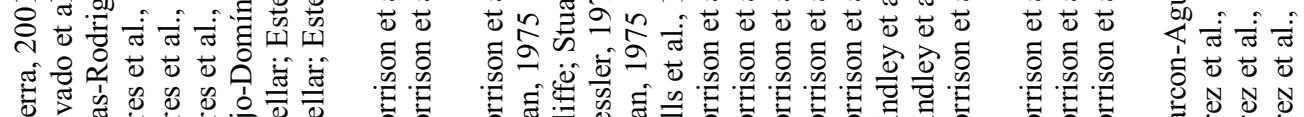

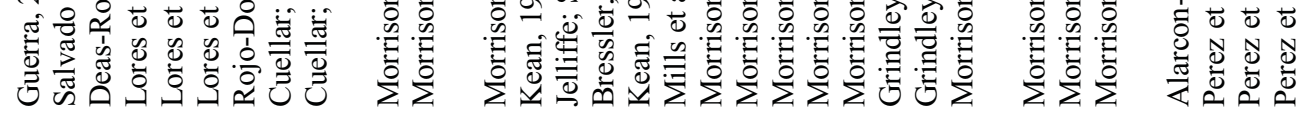

$\hat{a}$

ì

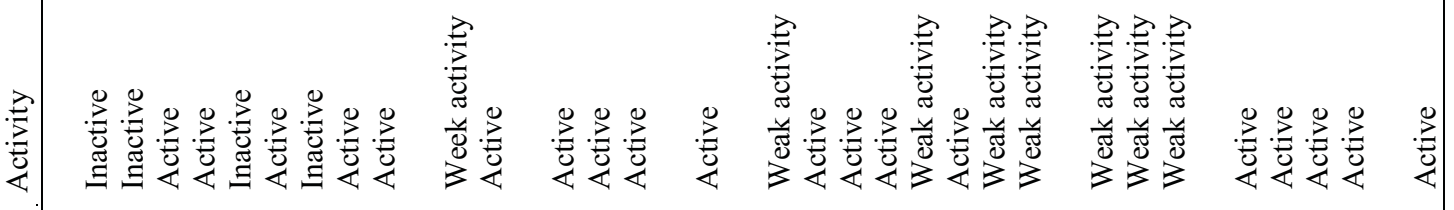

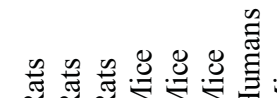

番.

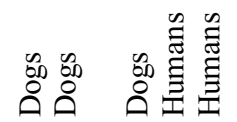

气ै

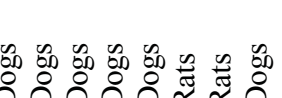

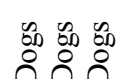

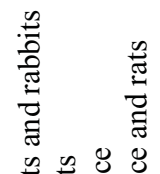

节

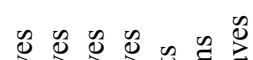

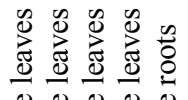

is

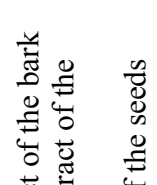

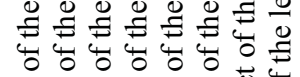

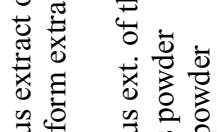

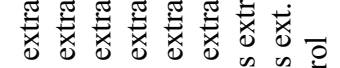

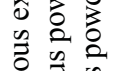

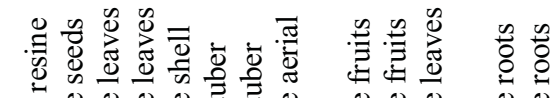

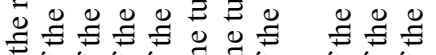

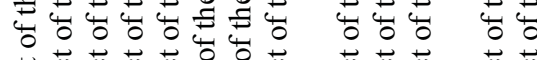

$\varangle$
0
0
0

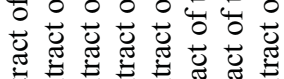

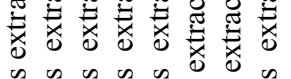

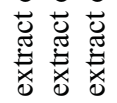

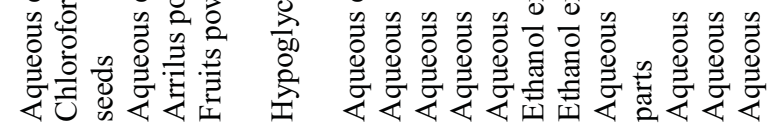

을 号

汹之

里

表

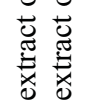

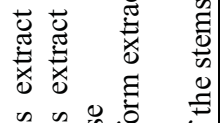

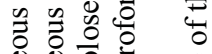

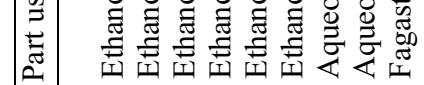

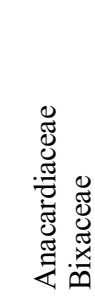

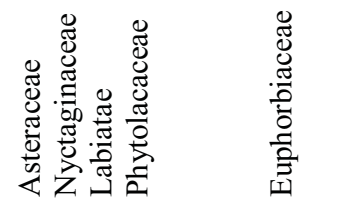

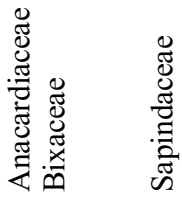
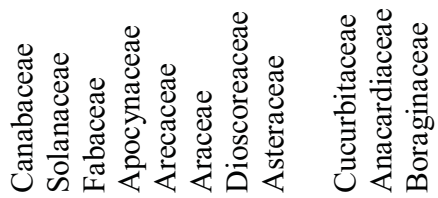

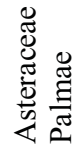
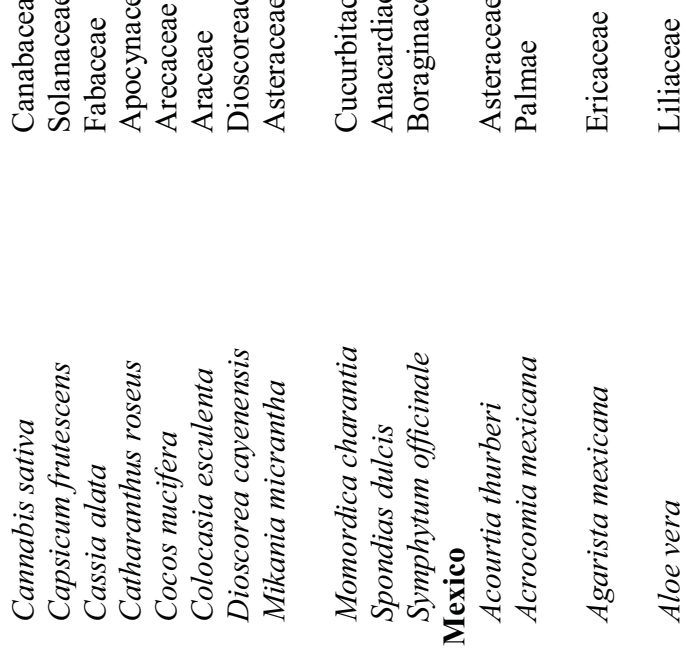


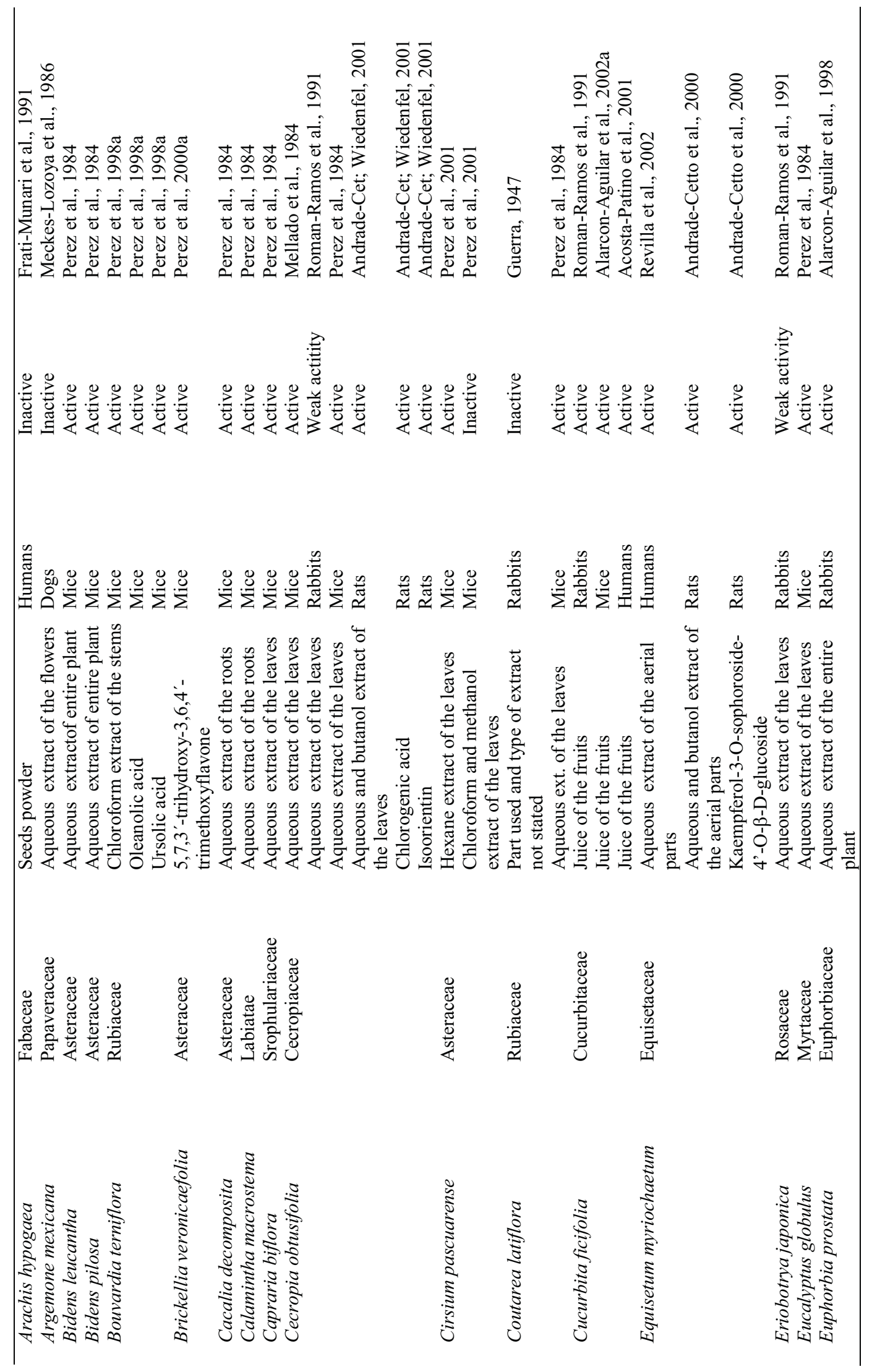




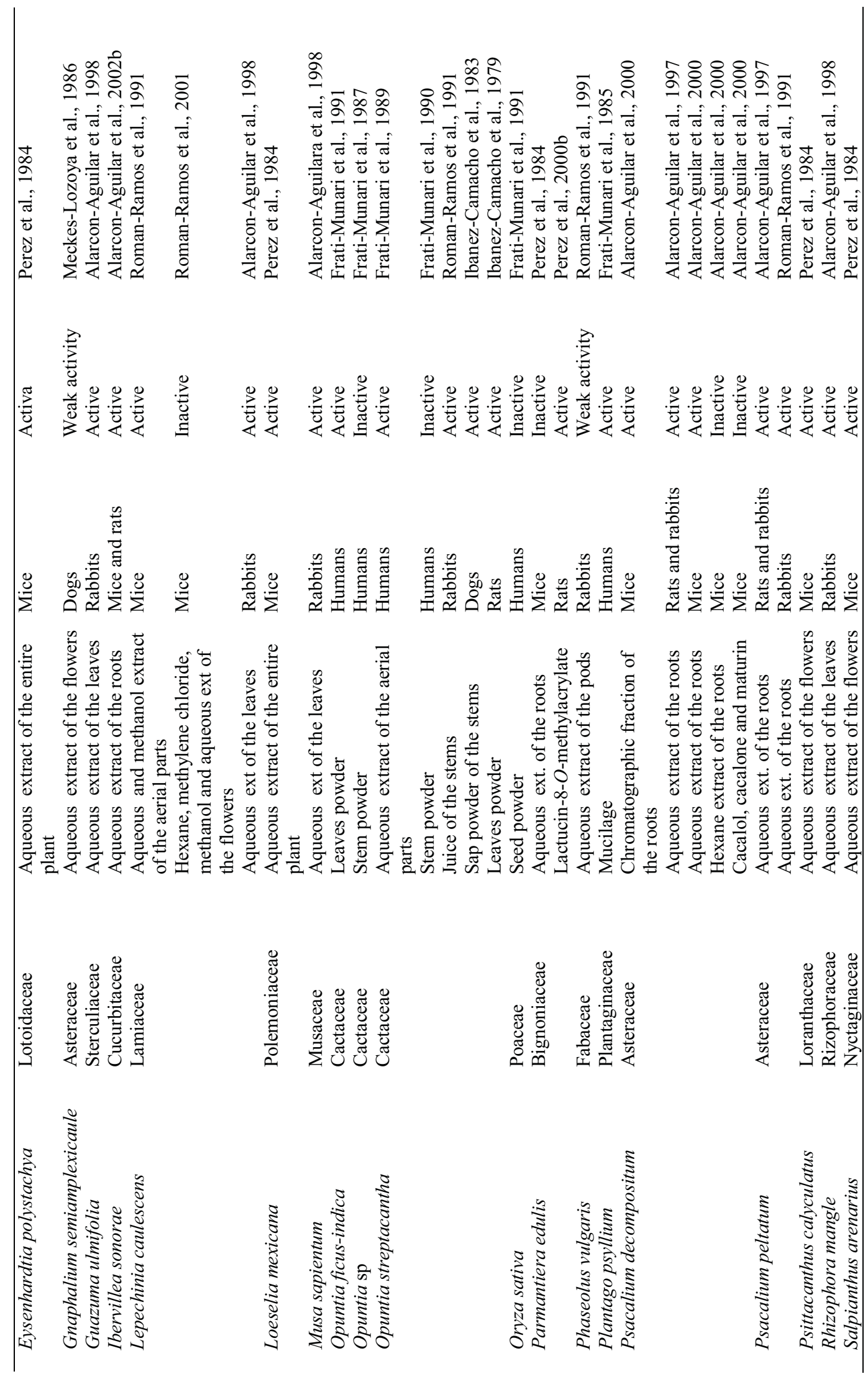




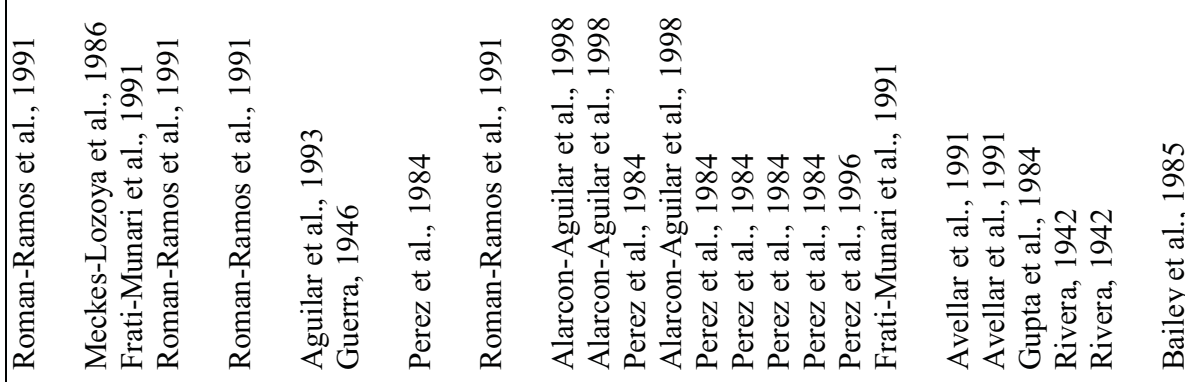

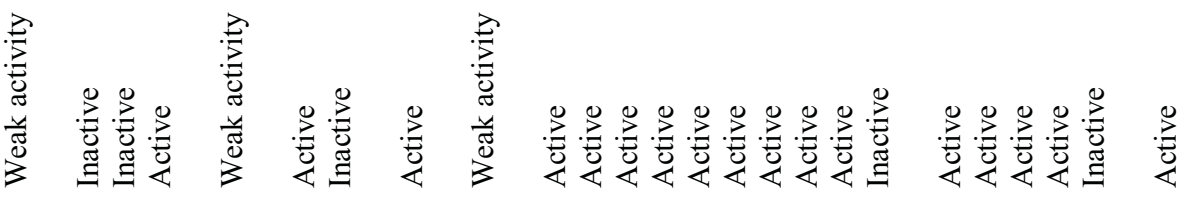

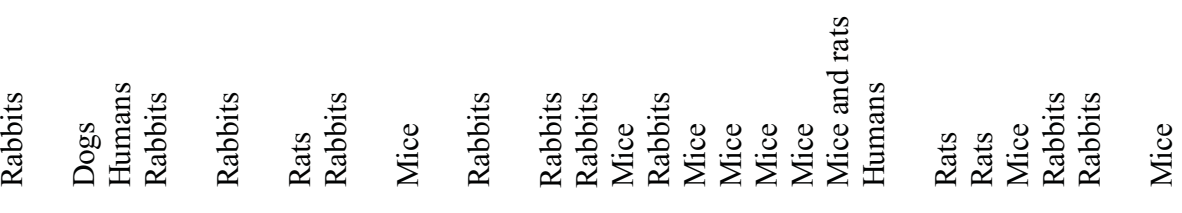

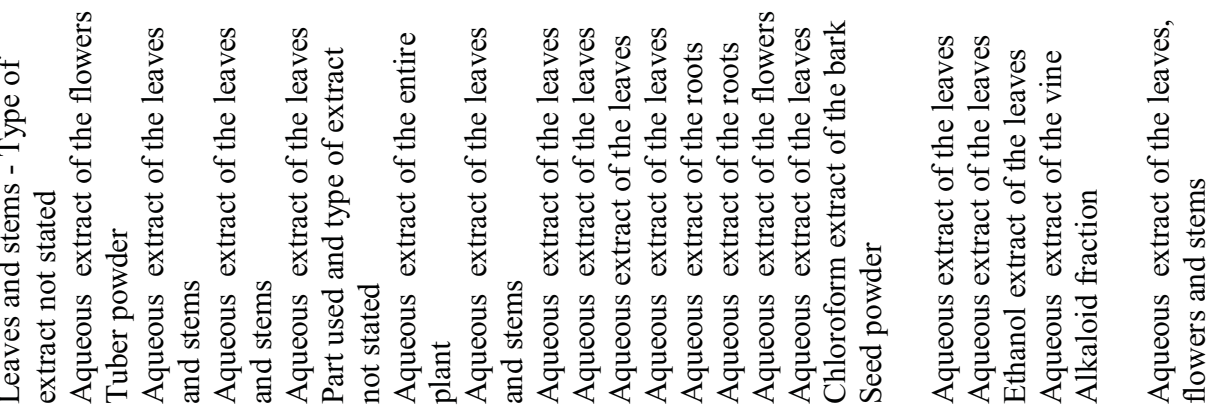

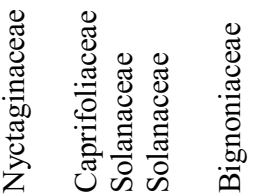

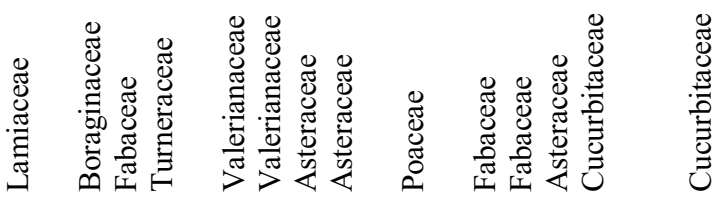

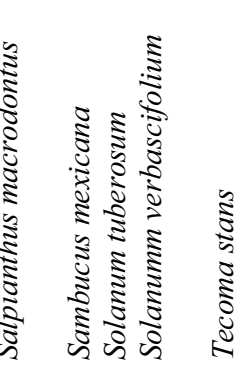

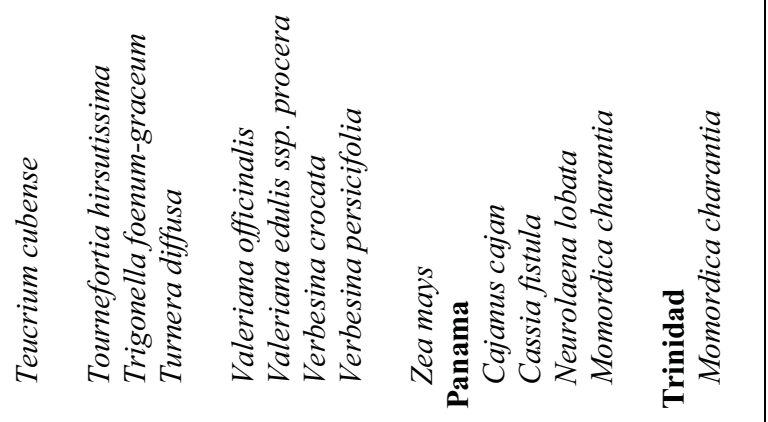




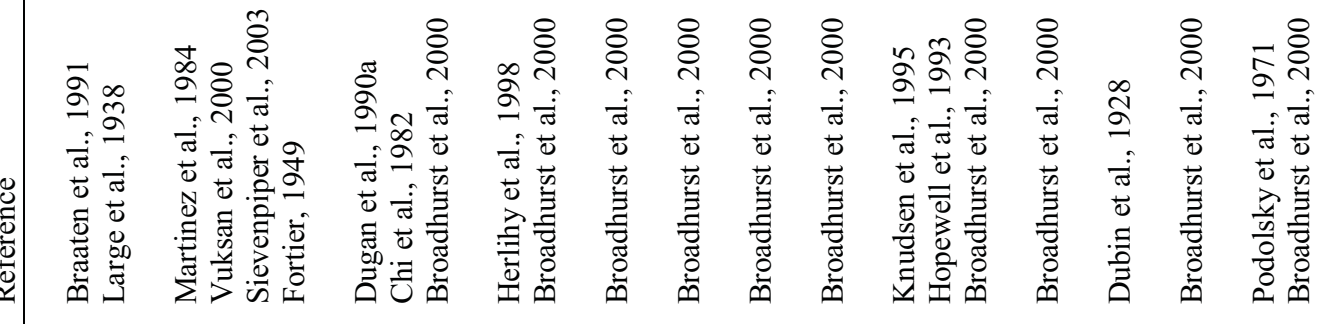

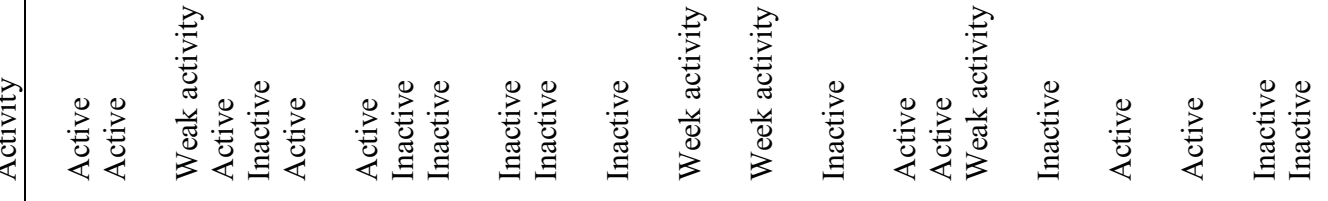

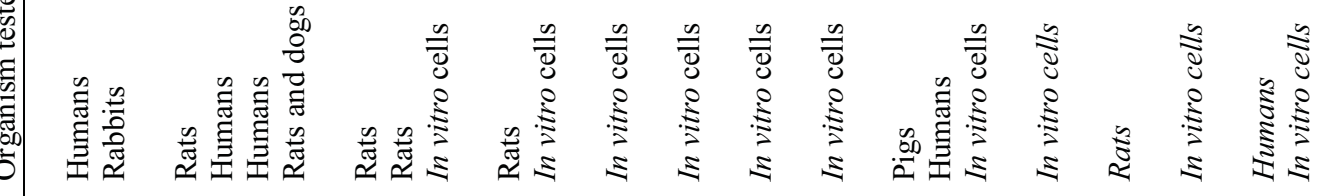

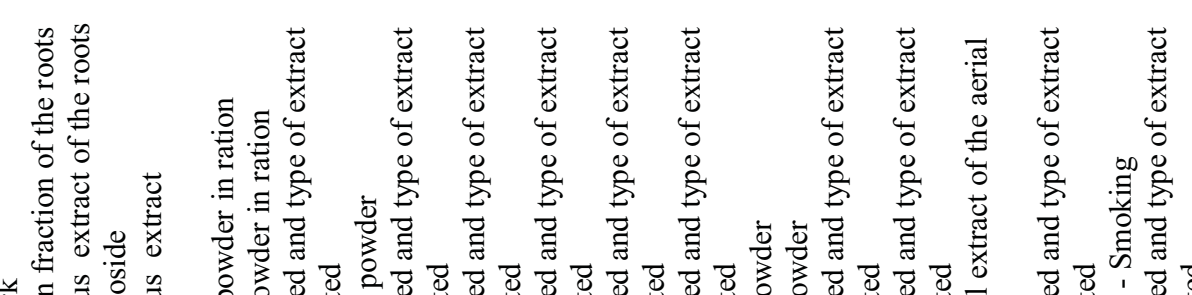

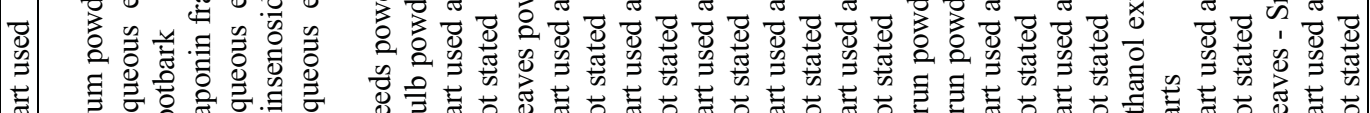

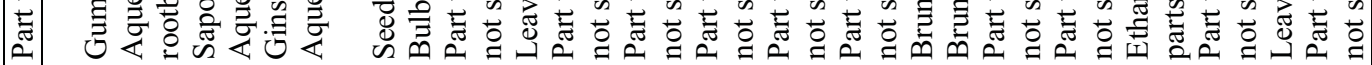

\section{政}




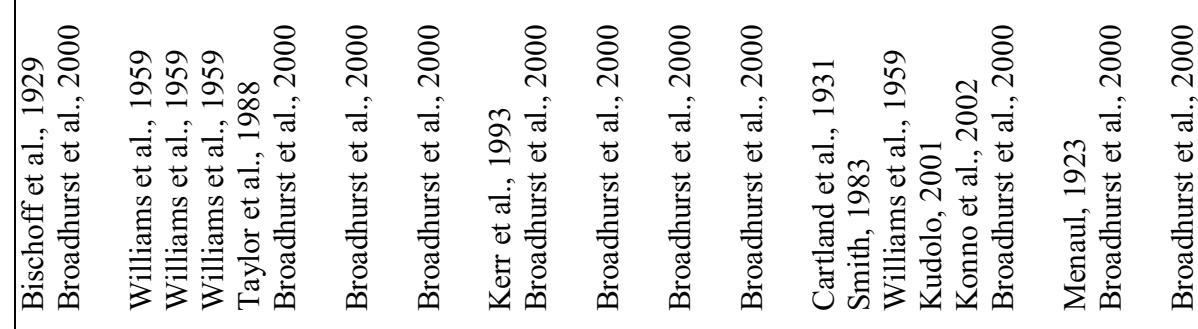

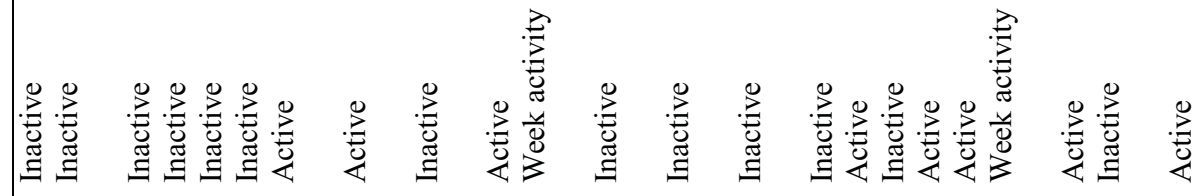

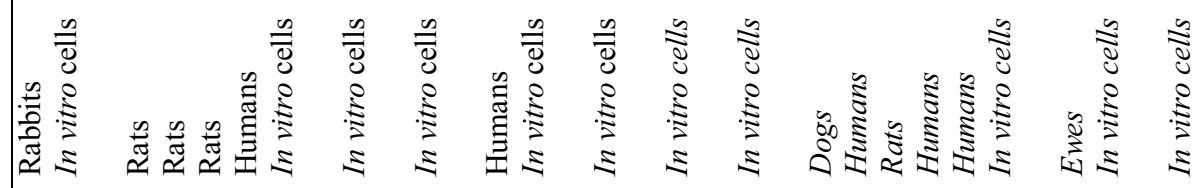

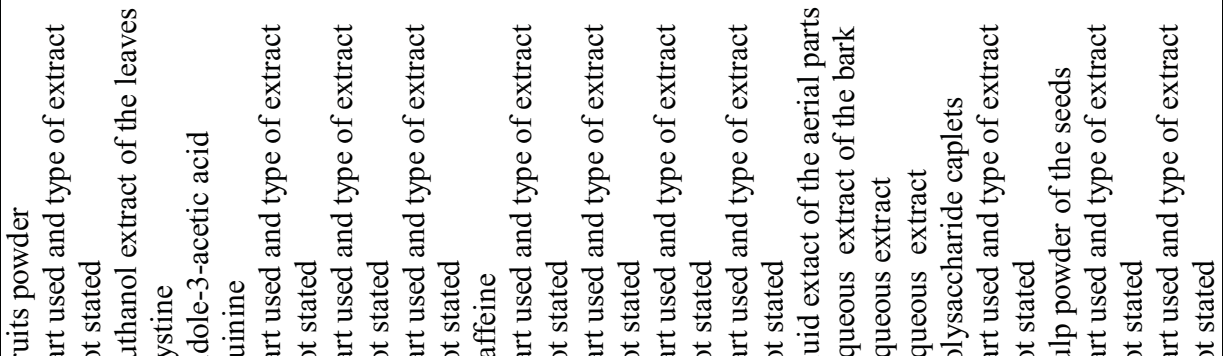

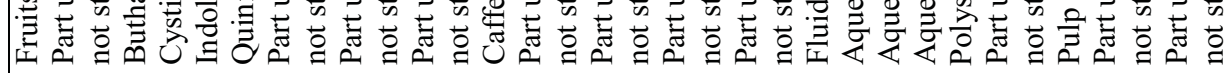

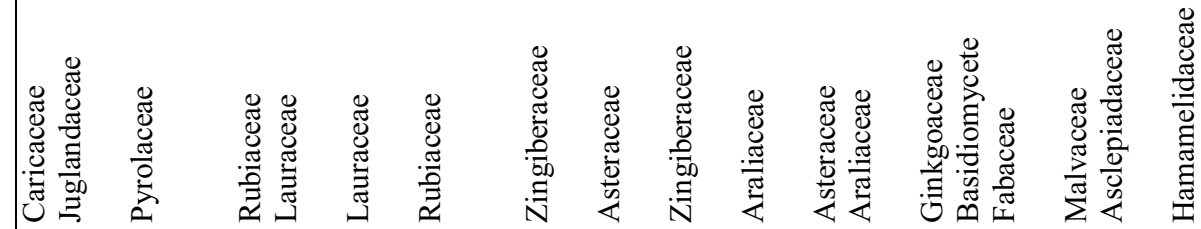

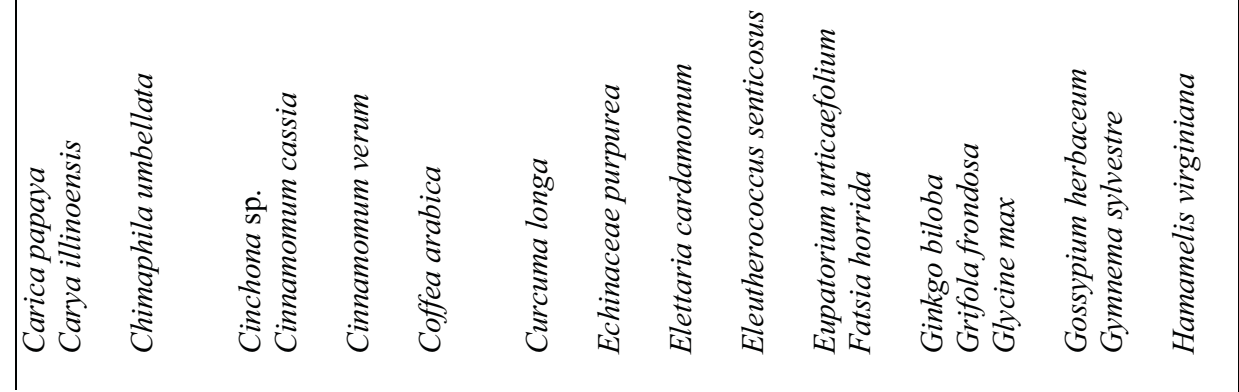




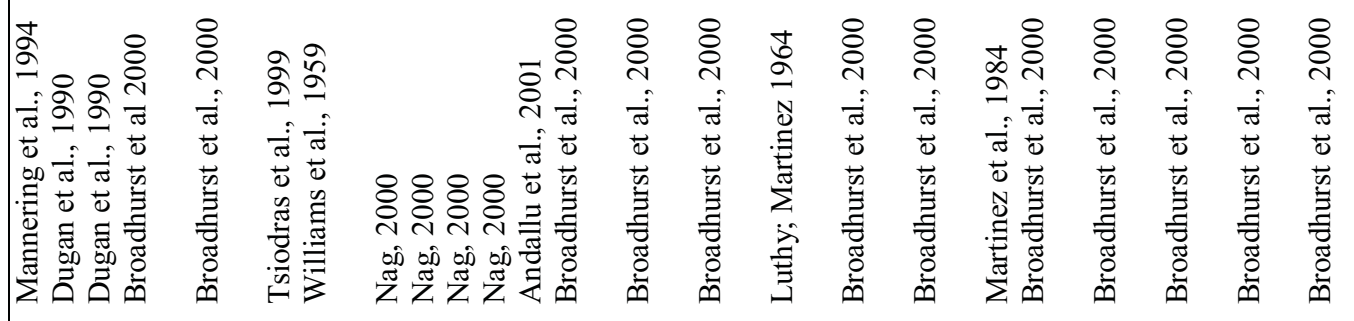

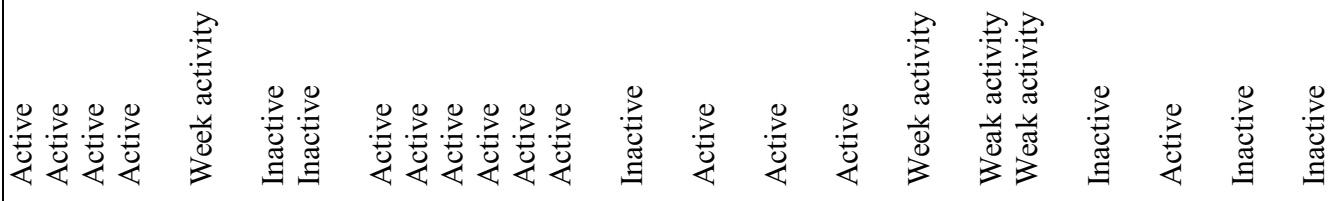

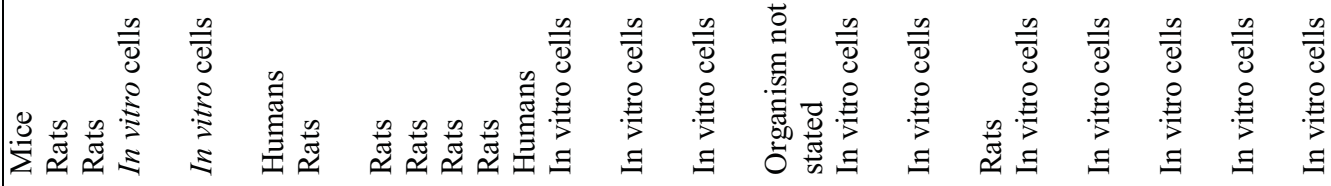

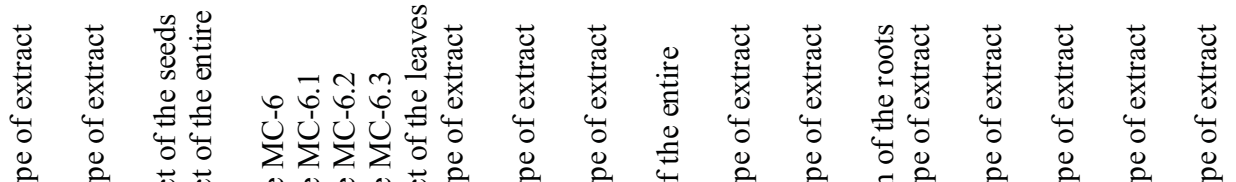

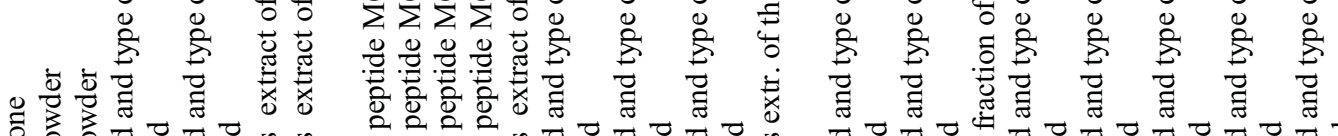

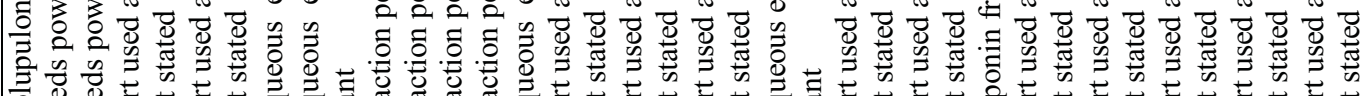

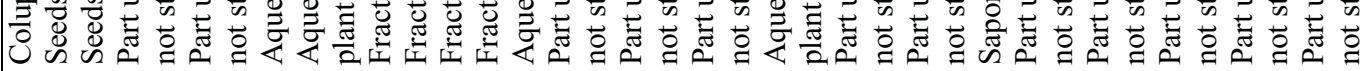

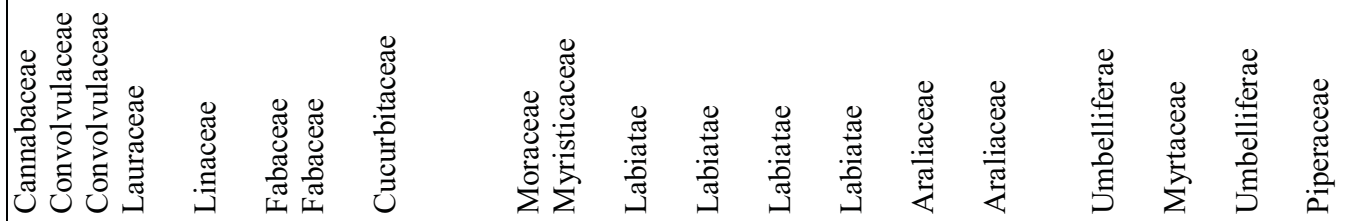

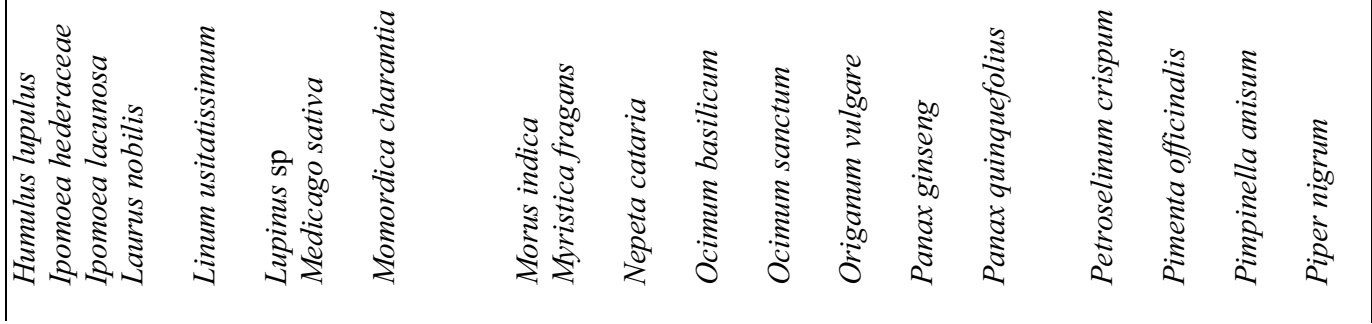




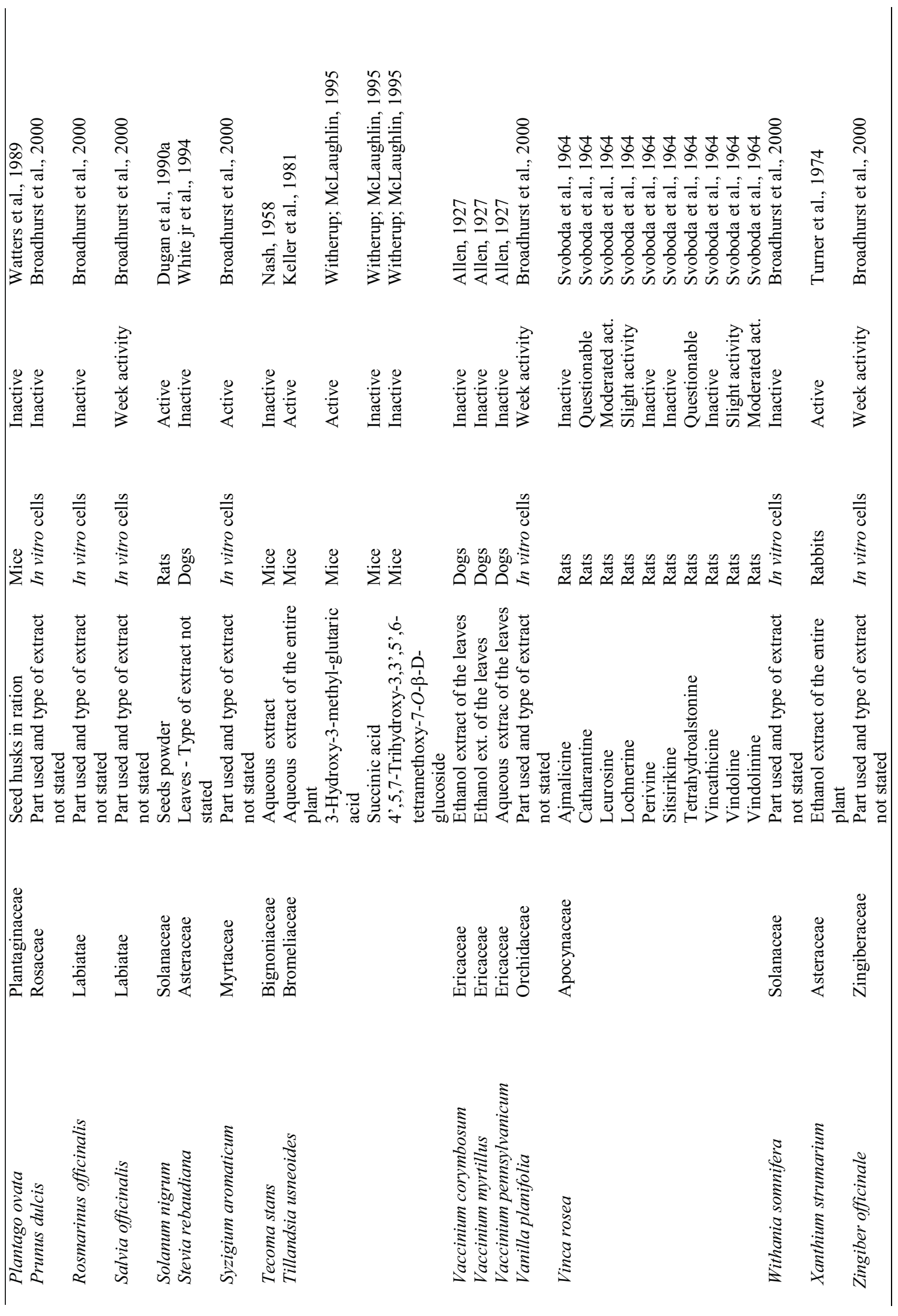




\section{REFERENCES}

Acosta-Patino JL, Jimenez-Balderas E, Juarez-Oropeza MA, Diaz-Zagoya JC 2001. Hypoglycemic action of Cucurbita ficifolia on Type 2 diabetic patients with moderately high blood glucose levels. $J$ Ethnopharmacol 77: 99-101.

Aguilar LC, Macias S, Chagoya A, Cardenas A, Diaz P, Cantu JM 1993. Antidiabetic activity of Tecoma stans in rats Fitoterapia 64: 304.

Alarcon-Aguilar FJ, Roman-Ramos R, Jimenez-Estrada M, Reyes-Chilpa B, Gonzalez-Paredes, Flores-Saenz JL 1997. Effects of three Mexican medicinal plants (Asteraceae) on blood glucose levels in healthy mice and rabbits. $J$ Ethnopharmacol 55: 171-77.

Alarcon-Aguilar FJ, Roman-Ramos R, Perez-Gutierrez S, Aguilar-Contreras A, Contreras-Weber CC, FloresSaenz JL 1998. Study of the anti-hyperglycemic effect of plants used as antidiabetics. $J$ Ethnopharmacol 61: 101-110.

Alarcon-Aguilar FJ, Jimenez-Estrada M, Reyes-Chilpa R, Roman-Ramos R 2000. Hypoglycemic effect of extracts and fractions from Psacalium decompositum in healthy and alloxan-diabetic mice. J Ethnopharmacol 72: 21-27

Alarcon-Aguilar FJ, Hernández-Galicia E, Campos-Sepulveda AE, Xolalpa-Molina S, Rivas-Vilchis JF, VasquezCarrillo LI, Roman-Ramos R 2002a. Evaluation of the hypoglycemic effect of Cucurbita ficifolia Bouche (Curcurbitaceae) in different experimental models. J Ethnopharmacol 82: 185-189.

Alarcon-Aguilar FJ, Campos-Sepulveda AE, Xolalpa-Molina S, Hernández-Galicia E, Roman-Ramos R 2002b. Hypoglycaemic activity of Ibervillea sonorae roots in healthy and diabetic mice and rats. Pharma Biol 40: 570-575.

Alarcon-Aguilar FJ, Roman-Ramos R, Flores-Saenz JL, Aguirre-Garcia F 2002c. Investigation on the hypoglycaemic effects of extracts of four Mexican medicinal plants in normal and alloxan-diabetic mice Phytother Res 16: 383-386.

Allen FM 1927. Blueberry leaf extract physiologic and clinical properties in relation to carbohydrate metabolism. $J$ Am Med Ass 89: 1577-1581.

Almeida RN, Barbosa-Filho JM, Naik SR 1985. Chemistry and pharmacology of an ethanol extract of Bumelia sartorum. J Ethnopharmacol 14: 173-185.

Almeida RN, Agra MF 1986. Levantamento bibliográfico da flora medicinal de uso no tratamento da diabetes e alguns resultados experimentais. Rev Bras Farm 67: $105-110$

Almeida RN, Navarro DS, Barbosa-Filho JM 2001. Plants with central analgesic activity Phytomedicine 8: 310322.

Alvares M, Bazzone RB, Godoy GL, Cury R, Botion LM 1981. Hypoglycemic effect of Stevia rebaudiana Bertoni I Brazilian Seminar on Stevia rebaudiana 25-26.

Andallu B, Suryakantham V, Srikanthi BL,. Reddy GK 2001. Effect of mulberry (Morus indica L.) therapy on plasma and erythrocyte membrane lipids in patients with type 2 diabetes. Clin Chim Acta 314: 47-53.

Andrade-Cetto A, Wiedenfeld H, Revilla MC, Sergio IA 2000. Hypoglycemic effect of Equisetum myriochaetum aerial parts on streptozotocin diabetic rats. $J$ Ethnopharmacol 72: 129-133.

Andrade-Cetto A, Wiedenfeld H 2001. Hypoglycemic effect of Cecropia obtusifolia on streptozotocin diabetic rats. J Ethnopharmacol 78: 145-149.

Arduino F, Soares MLNG 1951. Hypoglycemic action of Anacardium occidentale (Cashew) in normal individuals Brazil Med 65: 305-308.

Araújo IML 1999. Avaliação do efeito hipoglicemiante da Citrullus vulgaris Schrad (Melancia) em individuos diabéticos tipo 2 e normais. João Pessoa, 188p. Dissertação de Mestrado. Universidade Federal da Paraíba.

Avellar ME, Díaz A, Garcia I 1991. Evaluation de la medicina tradicional: efectos de Cajanus cajan L. (Guandu) y de Cassia fistula L. (Canafistula) en el metabolismo de los carbohidratos en el ratón. Rev Méd Panamá 16: 39-45.

Aybar MJ, Riera ANS, Grau A, Sanchez SS 2001. Hypoglycemic effect of the water extract of Smallantus sonchifolius (yacon) leaves in normal and diabetic rats. $J$ Ethnopharmacol 74: 125-132.

Bailey CJ, Day C 1989. Traditional plant medicines as treatments for diabetes. Diabetes Care 12: 553-564.

Bailey CJ, Day C, Turner SL, Leatherdale BA 1985. Cerasee, a traditional treatment for diabetes. Studies in normal and streptozotocin duabetic mice. Diabetes Res 2 : 81-84.

Barbosa WLR, Santos WRA, Pinto LN, Tavares ICC 2002. Flavonóides de Cissus verticillata e a atividade hipoglicemiante do chá de suas folhas. Rev Bras Farmacogn 12(Supl 1): 13-15.

Barceló A, Aedo C, Rajpathak S, Robles S 2003. The cost of diabetes in Latin America and the Caribbean. Bull WHO 81: 19-27.

Basnet P, Kadota S, Terashima S, Shimizu M, Namba T 1993. Two new 2-arylbenzofuran derivatives from hypoglycemic activity-bearing fractions of Morus insignis. Chem Pharm Bull. 41: 1238-1243.

Beltrame FL, Sartoretto JL, Bazotte RB, Cuman RN, Cortez DAG 2001. Phytochemical study and evaluation of the antidiabetic potential of Cissus sicydides L. (Vitaceae). Quím Nova 24: 783-785.

Bischoff F, Long ML, Sahyun M 1929. Investigations of the hypoglucemic properties of reglykol pancreapatine and papaw. J Pharmacol Exp Ther 36: 311-312.

Braaten JT, Wood PJ, Scott FW, Riedel KD, Poste LM, Collins MW 1991. Oat gum lowers glucose and insulin after an oral glucose load. Amer J Clin Nutr 53: $1425-1430$

Bresciani LFV, Yunes RA, Burger C, De Oliveira LE, Bof KL, Cechinel V 2004. Seasonal variation of kaurenoic acid, a hypoglycemic diterpene present in Wedelia paludosa (Acmela brasiliensis) (Asteraceae). Z. Naturforschung C 59: 229-232.

Bressler R 1976. The unripe akee - forbidden fruit. $N$ Engl $J$ Med 295: 500-501.

Broadhurst CL, Polansky MM, Anderson RA 2000. Insulin-like biological activity of culinary and medicinal plant aqueous extracts in vitro. J Agric Food Chem 48: 849-852.

Camargo MF, Lacerda AJB, Teixeira IPB, Martins DTO, Kawashita NH (2002). Efeito do tratamento de ratas 
diabéticas com extrato bruto da planta Echinodorus macrophyllys Micheli (Chapéu-de-couro). XVII Simpósio de Plantas Medicinais do Brasil, Cuiabá, MT, Brazil.

Cardoso FV, Andrade NED, Lima ACSF, Longhi DT, Brigido AO, Marcucci MC,.Scremin A, Paulino NI 2002. Avaliação da atividade dos extratos de plantas utilizadas na medicina popular para o tratamento da diabetes, sobre os canais de potássio modulados por ATP (KATA). XXXIV Congresso Brasileiro de Farmacologia e Terapêutica Experimental, Águas de Lindóia, SP, Brazil, p.215.

Cartland, GF, Heyl FW,.Neupert EF 1931. The hypoglycemic properties of white snakeroot (Eupatorium urticaefolium). J Amer Pharm Ass 20: 448-449.

Carvalho ES, Araújo KGL, Rocha L, Lúcio EMRA, Sharapin N, Kaplan MAC 2002. Efeito dos extratos metanólico e aquoso das partes aéreas de Bidens pilosa L. em alfa-glicosidase. XVII Simpósio de Plantas Medicinais do Brasil, Cuiabá, MT, Brazil, FT.136.

Chi MS, Koh ET, Stewart TJ 1982. Effects of garlic on lipid metabolism in rats fed cholesterol or lard. $J$ Nutr 112: 241-248.

Cuellar A, Estévez P 1980. A phytochemical study of Cuban plants. Rev Cubana Farm 14: 63-68.

Damasceno DC, Volpato GT, Calderon I.MP, Rudge MVC 2002a. Estudos dos extratos de folhas de Averrhoa carambola e Eugenia jambolana, obtidas em farmácia de manipulaçäo, sobre o diabete experimental. Rev Bras Toxicol 15: 9-14.

Damasceno DC, Lima PHO, Galhiane MS, Volpato GT, Rudge MVC 2002b. Avaliação do efeito hipoglicemiante da sapogenina extraida de sementes de Eugenia jambolana Lam. Rev Bras Pl Med 4: 46-54.

De Aguiar JC, Lins LJC 1958. Hypoglycemic action of inner bark (bast) of cashew tree (Anacardium occidentale). I. Action of a decoction on normal rats. Anais Fac Med Univ Recife 18: 193-197.

Deas-Rodriguez M, Menéndez R, Alvarez A, González R (1988) Efecto hipoglicemiante de la albahaca morada / Hypoglycemic effect of Ocymun Sanctum L Rev Cuba Invest Bioméd 7: 53-9

Deas-Rodriguez M, Seuc-Jo A, González-Suárez RM 1997. Estudio del efecto hipoglicemiante del Ocimun sanctum L. (albahaca-morada) con el uso de un ensayo biológico en ratones. Rev Cuba Plantas Med 2: $15-18$.

Derivi SCN, Mendez MHM, Rodrigues MCR, Fernandes ML, Silva MF 1987. Ação da fibra solúvel - pectina sobre os níveis glicêmicos em ratos. Rev Bras Farm 68: $1-7$.

Diaz J, Durruty P, Tapia JC, Carrasco E, Riesco V, Durruty G, Garcia-de-los-Rios M 1990. Effect of dietary fiber in patients with noninsulin dependent diabetesmellitus. Rev Med Chil 118: 24-32.

Dubin HE, Corbitt HB 1928. Hypoglucemia-producing substance. Patent US 1,653,452.

Dugan GM, Gumbmann MR 1990a. Toxicological evaluation of sicklepod and black nightshade seeds in shortterm feeding studies in rats. Food Chem Toxicol 28: 101-107.

Dugan GM, Gumbmann MR 1990b. Toxicological evaluation of morning glory seed: subchronic 90-day feeding study. Food Chem Toxicol 28: 553-559.

Dugan GM, Gumbmann MR 1990a. Toxicological and nutritional evaluation of velvetleaf seed: subchronic 90-day feeding study and protein efficiency ratio assay. Food Chem Toxicol 28: 95-99.

Ernest E 1997. Plants with hypoglycemic activity in humans. Phytomedicine 4: 73-78.

Farias RAF, Rao VSN, Viana GSB, Silveira ER, Maciel MAM, Pinto AC 1997. Hypoglycemic effect of transdehydrocrotonin, a nor-clerodane diterpene from Croton cajucara. Planta Med 63: 558-560.

Ferro E, Schinini A, Maldonado M, Rosner J, SchmedaHirschmann G 1988. Eugenia uniflora leaf extract and lipid metabolism in cebus apella monkeys. $J$ Ethnopharmacol 24: 321-325.

Fortier G 1949. Antidiabetic properties of Rhus typhina. Naval Med 14: 477-506.

Frati-Munari AC, Castillo-Insunza MR, Riva-Pinal H, ArizaAndraca CR, Banales-Ham M 1985. Effect of Plantago psyllium mucilage on the glucose tolerance test. Arch Invest Med (Mex) 16: 191-197.

Frati-Munari AC, Yever-Garces A, Islas-Andrade S, ArizaAndraca CR, Chavez-Negrete A 1987. Studies on the mechanism of hypoglycemic effect of nopal (Opunita sp.). Arch Invest Med (Mex) 18: 7-12.

Frati-Munari, AC, Altamirano BE, Rodriguez-Barcenas N, Ariza-Andraca R, Lopez-Ledesma R 1989. Hypoglucemic effect of Opuntia streptacantha Lemaire: research with crude extracts. Rev Archiv Invest Med (Mex) 20: 321-322.

Frati-Munari, AC, Gordillo BE, Altamirano P, Ariza CR, Cortes-Franco R, Chavez-Negrete A 1990. Acute hypoglycemic effect of Opuntia streptacantha Lemaire in niddm. Diabetes Care 13: 455-456.

Frati-Munari AC, Roca-Vides R, Lopez-Perez RJ, De-Vivero I, Ruiz-Velazco M 1991. Glycemia index of several foods in Mexico. Gac Med Mex 127: 163-171.

Galletto R, Siqueira VLD, Silva GEC, Nascimento KF, Bazotte RB 2003. XXXV Congresso Brasileiro de Farmacologia e Terapêutica Experimental, Águas de Lindóia, SP, Brazil, p. 203.

Gallo FN 1941. Action of extract of Bauhinia candicans on normal blood sugar and experimental diabetes of dogs. Rev Soc Argent Biol 17: 128.

Gómez R, Cervi FL, Guntzel C, Maslinkiewicz A, Barros HMT 2000. Curva glicêmica e variação de peso pelo extrato de erva de passarinho (Phrygilanthus acutifolius) em ratos. XV Reunião Anual da Federação de Sociedades de Biologia Experimental, Caxambu, MG, Brazil, p. 217.

Gonalons GP, Fontana A 1926. Effect of Phyllanthus sellowianus on blood-sugar concentration. Arch Argent Enferm Apar Digest Nutr 993.

Gonçalves MCR, Moura LSA, Rabelo LA, Barbosa-Filho JM, Cruz HMM, Cruz J 2000. Produtos naturais inibidores da enzima HMG CoA redutase. Rev Bras Farm 81: 63-71.

Gonzalez-Mujica F, Motta N, Márquez AH, Capote-Zulueta J 2003. Effects of Bauhinia megalandra aqueous leaf extract on intestinal glucose absorption and uptake by enterocyte brush border membrane vesicles. Fitoterapia 74: 84-90.

Grindley PBA, Omoruyi F, Asemota HN, Morrison EYSA 2002. 
Carbohydrate digestion and intestinal ATPases in streptozotocin-induced diabetic rats fed extract of yam (Dioscorea cayenensis) or dasheen (Colocasia esculenta). Nutri Res 22: 333-341.

Grover JK, Yadav S, Vats V 2002. Medicinal plants of India with anti-diabetic potential. J Ethnopharmacol 81: 81-100.

Guerra F 1946. The pharmacology of mexican antidiabetic plants. I. Action of Tecoma mollis on normal and diabetic blood sugar levels. Rev Inst Salubridad y Enfermedad Trop (Mex) 7: 237-248.

Guerra F 1947. The pharmacology of Mexican antidiabetic plants. II. Action of Coutarea latiflora on normal and diabetic blood-sugar levels. Rev Inst Salubridad y Enfermedad Trop (Mex) 8: 29-38.

Guerra RLL, Padton MCV, Rivas R, Blanco JCC, Parets MH, Gallardo AIA 2001. Efectos de un extracto hidroalcoholico de Bidens alba en ratas normales y con diabetes aloxanica. Acta Farm Bonaerense 20: 89-93.

Gupta MP, Solis NG, Esposito-Avella M, Sanchez C 1984. Hypoglycemic activity of Neurolaena lobata (1.) R.Br. J Ethnopharmacol 10: 323-327.

Handa SS, Chawla AS 1989. Hypoglycaemic plants - A review. Fitoterapia 60: 195-224.

Herlihy JT, Kim JD, Kalu DN, Nelson JF, Ward WF, Ikeno Y, Yu BP 1998. Effects of Aloe vera ingestion in the rat. II. Hormonal and metabolic characteristics. Phytother Res 12: 355-360.

Hnatysyzn O, Mino J, Gorzalczany S, Ferraro G, Coussio J, Acevedo C 1997. Antidiabetic activity of Phyllanthus sellowianus in streptozotocin-induced diabetic rats. Phytomedicine 4: 251-253.

Hnatyszyn O, Mino J, Ferraro G, Acevedo C 2002. The hypoglycemic effect of Phyllanthus sellowianus fractions in streptozotocin-induced diabetic mice. Phytomedicine 9: 556-559.

Hopewell R, Yeater R, Ullirich I 1993. Soluble fiber: effect on carbohydrate and lipid metabolism. Progress Food Nutr Sci 17: 159-182.

Ibanez-Camacho R, Meckes-Lozoya M, Mellado-Campos V 1983. The hypoglucemic effect of Opuntia streptacantha studied in different animal experimental models. J Ethnopharmacol 7: 175181.

Ibanez-Camacho R, Roman-Ramos R 1979. Hypoglycemic effect of Opuntia cactus. Arch Invest Med (Mex) 10: 223-230.

Ishii EL, Bracht A 1985. Açäo do isosteviol e do steviol sobre o metabolismo de carboidratos em fígado de rato perfundido isoladamente. Rev Unimar 7: 97-121

Ivorra MD, Paya M, Villar A 1989. A review of natural products and plants as potential anti-diabetic drugs. $J$ Ethnopharmacol 27: 243-275.

Jelliffe DB, Stuart KL 1954. Acute toxic hypoglycaemia in the vomiting sickness of Jamaica. Brit Med J 1: 75-77.

Juliant C. 1931. The hypoglucemic action of unha de vacca (Bauhinia fortificats). Rev Sud Amer Endocrinol Immunol Quimioter 14: 325-327.

Kanegusuku M, Benassi JC, Pedrosa RC, Yunes RA, CechinelFilho V, Maia AA, .Souza MM, Delle-Monache F, Niero R 2002. Cytotoxic, hypoglycemic activity and phytochemical analysis of Rubus imperialis
(Rosaceae). Z Naturforschung C 57: 272-276.

Kean EA 1975. Hypoglycin. Academic press, New York.

Keller WJ, Bourn WM, Bonfiglio JFA 1981. A folk medicine for diabetes mellitus. Q J Crude Drug Res 19: 49-51.

Kerr D, Sherwin RS, Pavalkis F, Fayad PB, Sikorski L, Rife F, Tamborlane WV, During MJ 1993. Effect of caffeine on the recognition of and responses to hypoglycemia in humans. Ann Intern Med 119: 799-804.

Knudsen KEB, Johansen HN 1995. Mode of action of oat bran in the gastrointestinal tract. Eur J Clin Nutr 49: S163-S169.

Kono S, Aynehchi S, Dolin DJ, Schwartz AM, Choudhury MS, Tazaki H 2002. Anticancer and hypoglycemic effects of polysaccharides in edible and medicinal maitake mushroom (Grifola frondosa (Dicks.: Fr.) S. F. Gray). Inter J Med Mushrooms 4: 185-195.

Krenisky JM, Luo J, Reed MJ, Carney JR 1999. Isolation and antihyperglycemic activity of bakuchiol from Otholobium pubescens (Fabaceae), a peruvian medicinal plant used for the treatment of diabetes. Biol Pharm Bull 22: 1137-1140.

Kudolo GB 2001. The effect of 3-month ingestion of Ginkgo biloba extract (EGb 761) on pancreatic betacell function in response to glucose loading in individuals with non-insulin-dependent diabetes mellitus. J Clin Pharmacol 41: 600-611.

Lamba SS, Buch KY, Lewis H, Lamba HJ 2000. Phytochemicals as potential hypoglycemic agents. Studies in Natural Products Chemistry, 21: 457-495.

Large RG, Brocklesby HN 1938. A hypoglycemic substance from the roots of the devil's club (Fatsia horrida). Can Med Ass J 39: 32-35.

Lemus I, Garcia R, Jabsa Z, Erazo S, Garcia H 1986. Hypoglycemic activity of an extract of Bauhinia candicans B. Plant Med Phytother 20: 8-17.

Lemus I, Garcia R, Delvillar E, Knop G 1999. Hypoglycaemic activity of four plants used in Chilean popular medicine. Phytother Res 13: 91-94.

Longhi DT, Ballmann JD, Brigido AO, Marcucci MC, Scremin A, Paulino N 2003. Mechanisms involved in the hypoglicemic effect of ethanolic extract of Polymnia sonchifolia Poepp. \& Endl. XXXV Congresso Brasileiro de Farmacologia e Terapêtica Experimental, Águas de Lindóia, SP, Brazil, p. 226.

Lores RI, Pujol MC 1990. Petiveria alliaceae L. (Anamu) Study of the hypoglycemic effect. Med Interne 28: 347-352.

Luthy N, Martinez-Fortun O 1964. A possible oral hypoglycemic factor in abahaca morada (Ocimum sanctum). Ohio J. Sci. 64: 223-224.

Mannering GJ, Shoeman JA, Shoeman DW 1994. Effects of colupulone, a component of hops and brewers-yeast, and chromium on glucose-tolerance and hepatic cytochrome-P450 in nondiabetic and spontaneously diabetic mice. Bioch Biophys Res Comm 200: 14551462.

Manrique JFM, Sousa YMA, Lima FO, Vasconcellos MC, Borras MRL, Ferreira LCL, Costa PRC, Roland IA 2002. Efeito do extrato aquoso de Brosimum acutifolium sobre os níveis plasmátaticos de glicose, colesterol e triglicerídeod em ratos wistar. XVII Reunião Anual da Federação de Sociedades 
de Biologia Experimental, Caxambu, MG, Brazil, p. 20.085 .

Marles RJ, Farnsworth NR 1995. Antidiabetic plants and their active constituents. Phytomedicine 2: 137-189.

Martinez B, Staba EJ 1984. The physiological effects of aralia, panax and eleutherococcus on exercised rats. Jap J Pharmacol 35: 79-85.

Matsumura T, Kasai M, Hayashi T, Arisawa M, Momose Y, Arai I, Amagaya S, Komatsu Y 2000. Alpha-glucosidase inhibitors from Paraguayan natural medicine, nangapiry, the leaves of Eugenia uniflora. Pharma Biol 38: 302-307.

Meckes-Lozoya M, Campos VM 1986. Pharmacological screening of Mexican plants, popularly used for the treatment of cough. Fitoterapia 57: 365-370.

Mellado V, Lozoya M 1984. Effect of the aqueous extract of Cecropia obtusifolia on the blood sugar of normal and pancreatectomized dogs. Int J Crude Drug Res 22: 11-16.

Menaul P. 1923. The physiological effect of gossypol. J Agr Res 26: 233-224.

Mills J, Melville GN, Bennett C, West M, Castro A 1987. Effect of hypoglycin-A on insulin release. Biochem Pharmacol 36: 495-497.

Miron VR, Mazzanti CM, Schossler DRC, Filappi A, Prestes D, Balz D, Morsch A, Cecim M, Schetinger MRC, Morsch VM, Neu TN 2002. Extrato da casca de Syzygium cumini na glicemia e estresse oxidativo de ratos normais e diabéticos. XVII Reunião Anual da Federação de Sociedades de Biologia Experimental, Caxambu, MG, Brazil, p. 16.008.

Morais LCSL, Barbosa-Filho JM, Almeida RN 2003. Plants and bioactives compounds for the treatment of Parkinson's desease. Arquivo de Fitomedicina 1: 127-132.

Morato GS, Calixto JB, Cordeiro L, De-Lima TCM; Morato EF, Nicolau M, Era GA, Takahashi RN, Valle RMR, Yunes RA 1989. Chemical and pharmacological studies on Talauma ovata St. Hil. (Magnoliaceae). J Ethnopharmacol 26: 277-286.

Mori T, Nishikawa Y, Takata Y, Kashiuchi N, Ishihara N 2001. Effect of insulina leaf extract on development of diabetes. Comparison between normal, streptozotocin-induced diabetic rats and hereditary diabetic mice. Nippon Eiyo Shokuryo Gakkaishi 54: 197-203.

Morrison EY, West ME 1982. A preliminary study of the effects of some West Indian medicinal plants on blood sugar levels in the dog. West Indian Med J 31: 194197.

Morrison EY, West ME 1985. The effect of Bixa orellana (annatto) on blood sugar levels in the anaesthetized dog. West Indian Med J 34: 38-42.

Moura MD, Torres AR, Oliveira RAG, Diniz MFFM, BarbosaFilho JM 2001. Natural products inhibitors of models of mammary neoplasia. Brit J Phytotherapy 5: 124-145.

Moura MD, Silva JS, Oliveira RAG, Diniz MFFM, BarbosaFilho JM 2002. Natural products reported as potential inhibitors of uterine cervical neoplasia. Acta Farm Bonaerense 21: 67-74.

Nag B 2000. Orally active fraction of Momordica charantia, active peptides thereof, and their use in the treatment of diabetes. Patent US 6,127,338 p.1-11.

Naik SR, Barbosa-Filho JM, Dhuley JN 1991. Probable mechanism of hypoglycemic activity of bassic acid, a natural product ilsolated from Bumelia sartorum. J Ethnopharmacol 33: 37-44.

Nash JB, Albers CC, Howard JK, Fly Jr SH 1958. Lack of antidiabetogenic and antidiabetic effects of Tecoma stans in oxan diabetes. Tex Rep Biol Med 8: 350353.

Neves RE, Menezes FS, Pereira NA, Moreira DL 2002. Avaliação farmacológica de plantas medicinais utilizadas como hipoglicemiantes na região do médio Paraíba do estado do Rio de Janeiro. XVII Simpósio de Plantas Medicinais do Brasil, Cuiabá, MT, Brazil, p. FT. 091.

Novaes EP, Rossi C, Poffo C, Pretti E, Oliveira AE, Schlemper V, Niero R, Cechinel-Filho V, Burger C 2001. Preliminary evaluation of the hypoglycemic effect of some Brazilian medicinal plants. Therapie 56: 427-430.

Oliveira ACP, Endringer DC, Coelho MM 2003. The starch from Solanum lycocarpum St. Hill. fruit is not a hypoglycemic agent. Braz J Med Biol Res 36: 525530.

Oliveira F, Saito ML 1989. Alguns vegetais brasileiros empregados no tratamento da diabetes. Rev Bras Farm 2/4: 170-196.

Oliveira AEA, Machado OLT, Gomes VM, Neto JX, Pereira AC, Vieira JGH, Fernandes KVS, Xavier J 1999. Jack bean seed coat contains a protein with complete sequence homology to bovine insulin. Protein and Peptide Letters 6: 15-21.

Ospina LF, Olarte JE, Calle J, Pinzón R 1995. Comprobación de la actividad hipoglicemiante y captadora de radicales libres oxigenados de los principios activos de Curatella americana L. Rev Colomb Ci Quim Farm 24: 6-11.

Oviedo CA, Fronciani G, Moreno R, Maas L 1970. Hypoglycemic action of Stevia rebaudiana. Excerpta Medica 209: 92-96.

Pepato MT, Oliveira JR, Kettelhut IC, Migliorini RH 1993. Assessment of the antidiabetic activity of Myrcia uniflora extracts in streptozotocin-diabetic rats. Diabetes Res 22: 49-57.

Pepato MT, Folgado VBB, Kettelhut IC, Brunetti IL 2001. Lack of antidiabetic effect of a Eugenia jambolana leaf decoction on rat streptozotocin diabetes. Braz $J$ Med Biol Res 34: 389-395.

Pepato MT, Keller EH, Baviera AM, Kettelhut IC, Vendramini RC, Brunetti IL 2002. Anti-diabetic activity of Bauhinia forficata decoction in streptozotocindiabetic rats J Ethnopharmacol 81: 191-197.

Pepato MT, Baviera AM, Vendramini RC, Perez MPMS, Kettelhutt IC, Brunett IL 2003. Cissus sicyoides (princess vine) in the long-term treatment of streptozotocin-diabetic rats. Biotechnol Appl Biochem 37(Part 1): 15-20.

Pereira NA 1997. Plants as hypoglycemic agents. Ciência e Cultura 49: 354-358.

Pereira JV, Modesto-Filho J, Agra MF, Barbosa-Filho JM 2002. Plant and plant-derived compounds employed in prevention of the osteoporosis. Acta Farm Bonaerense 21: 223-234. 
Perez RM, Ocegueda A, Muñoz JL, Ávila JG, Morrow WW 1984. A study of the hypoglucemic effect of some mexican plants. J Ethnopharmacol 12: 253-262.

Perez RM, Perez S, Zavala MA, .Perez C 1996. Effects of Agarista mexicana and Verbesina persicifolia on blood glucose level of normoglycaemic and alloxandiabetic mice and rats. Phytother Res 10: 351-353.

Perez RM, Zavala MA, Vargas SR 1997. Coyolosa, a new hypoglycemic from Acrocomia mexicana. Pharm Acta Helv 13: 105-111.

Perez RM, Perez C, Perez S, Zavala MA 1998a. Effect of triterpenoids of Bouvardia terniflora on blood sugar levels of normal and alloxan diabetic mice. Phytomedicine 5: 475-448.

Perez RM, Zavala MA, Perez S, Perez C 1998b. Antidiabetic effect of compounds isolated from plants. Phytomedicine 5: 55-75.

Perez RM, Cervantes H, Zavala MA, Sánchez SJ, Perez S, Perez C 2000a. Isolation and hypoglycemic activity of 5, 7,3'-trihydroxy-3,6,4'-trimethoxyflavone from Brickellia veronicaefolia. Phytomedicine 7: 25-29.

Perez RM, Perez C, Zavala MA, Perez S, Hernandez H, Lagunes F 2000b Hypoglycemic effects of lactucin8-O-methylacrylate of Parmentiera edulis fruit. $J$ Ethnopharmacol 71: 391-394.

Perez RM, Ramirez E, Vargas R 2001. Effect of Cirsium pascuarense on blood glucose levels of normoglycaemic and alloxan-diabetic mice. Phytother Res 15: 552-554.

Podolsky S, Pattavina CG, Amaral MA 1971. Effect of marijuana on the glucose-tolerance test. Ann N Y Acad Sci 191: 54-60.

Presta GA, Pereira NA 1987. Atividade de abageru, Chysobalanus icaco Lin. (Chrysobalanaceae) em modelos experimentais para o estudo de plantas hipoglicemiantes. Rev Bras Farm 68: 91-101.

Provasi M, Oliveira CE, Martinho MC, Pessini LG, Bazotte RB, Cortez DAG 2001. Avaliação da toxicidade e do potencial antihiperglicemiante da Averrhoa carambola L. (Oxalidaceae). Acta Sci. 23: 665669.

Rahman AU, Zaman K 1989. Medicinal-plants with hypoglycemic activity. $J$ Ethnopharmacol 26: 1-55.

Reis JC, Oliveira APO, Soares FR, Brito SMRC 2002. Avaliação da atividade hipoglicemiante de Vatairae macrocarpa (Benth) Ducke em ratos. XVII Reunião Anual da Federação de Sociedades de Biologia Experimental, Caxambu, MG, Brazil, p. 20.091.

Revilla MC, Andrade-Cetto A, Islas S, Wiedenfeld H 2002. Hypoglycemic effect of Equisetum myriochaetum aerial parts on type 2 diabetic patients. $J$ Ethnopharmacol 81: 117-120

Rivera G 1942. Preliminary chemical and pharmacological studies on "cundeamor", Momordica charantia. II. Amer J Pharm 114: 72-73.

Rocha LG, Almeida JRGS, Macedo RO, Barbosa-Filho JM 2005. A review of natural products with antileishmanial activity. Phytomedicine 12: 514-535.

Rodriguez J, Loyola C, Schmeda-Hirschmann G 1992. Hypoglycaemic activity of Hexachlamys edulis (yvahai) extract in rats. Phytother Res 6: 47-49.

Rodriguez J, Loyola JI, Maulen G, Schmeda-Hirschmann G 1994. Hypoglycaemic activity of Geranium core- core, Oxalis rosea and Plantago major extracts in rats. Phytother Res 8: 372-374.

Rojo-Domínguez D, Bell-Heredia L, Cansio-Martínez E, Iglesias-Lores R 2002. Efecto del extracto hipoglicemiante de Petiveria alliacea L sobre el consumo de glucosa por los eritrócitos. Rev Cuba Invest Bioméd 21: 161-166.

Roman-Ramos R, Flores-Saenz JL, Partida-Hernandez G, LaraLemus A, Alarcon-Aguilar FJ 1991. Experimental study of hypoglycemic activity of some antidiabetic plants. Arch Invest Med (Mex) 22: 87-93.

Roman-Ramos R, Contreras-Weber CC, Nohpal-Grajeda G, Flores-Saenz JL, Alarcon-Aguilar FJ 2001. Blood glucose level decrease caused by extracts and fractions from Lepechinia caulescens in healthy and alloxan-diabetic mice. Pharma Biol 39: 317-321.

Russo EMK, Reichelt AA, De-Sá JR, Furlanetto RP, Moises RC, Kasamatsu TS, Chacra AR 1990. Clinicaltrial of Myrcia uniflora and Bauhinia-forficata leaf extracts in normal and diabetic-patients. Braz J Med Biol Res 23: 11-20.

Salvado AC, Naranjo JP, Evseva EC, Royero RH, Rodríguez DLL 1997. Tamizaje, tecnologia, control de calidad y farmacologia del extracto fluido de Bouganvillea spectabilism Willd. Rev Cuba Plantas Med 2: 1925.

Sampaio EDM, Furtado FDAS, Furtado MJS, Cavalcante MNNDS, Riedel ODO 1979. Hypoglycemic effect of raw coffee beans (Coffea arabica L. Rubiaceae). Rev Med Univ Fed Ceara 19: 49-53.

Sievenpiper JL, Arnason JT, Leiter LA, Vuksan V 2003. Variable effects of American ginseng: A batch of American ginseng (Panax quinquefolius L.) with a depressed ginsenoside profile does not affect postprandial glycemia. Eur J Clin Nutr 57: 243-248.

Silva FRMB, Szpoganicz MG, Pizzolatti MA, Willrich V, Sousa E 2002. Acute effect of Bauhinia forficata on serum glucose levels in normal and alloxan-induced diabetic rats. J Ethnopharmacol 83: 33-37.

Silva JS, Moura MD, Oliveira RAG, Diniz MFFM, BarbosaFilho JM 2003. Natural products inhibitors of ovarian neoplasia. Phytomedicine 10: 221-232.

Silveira NA, Nascimento SDM, Ferreira JDJ, Carvalho OF, Paula JR 2001. Efeito do extrato etanólico de Rudgea viburnioides sobre a concentração plasmática de glicose, triglicérideos e colesterol em ratos Wistar. XVI Reunião Anual da Federação de Sociedades de Biologia Experimental, Caxambu, MG, Brazil, p. 318.

Smith GW 1983. Arctic Pharmacognosia II. Devil's club, Oplopanax horridus. J Ethnopharmacol 7: 313320.

Sousa E, Zanatta L, Charão CCT, Seifriz I, Creczynski-Pasa TB, Pizzolatti MG, Szpoganicz B, Silva FRMB 2002a. Efeito hipoglicêmico e propriedades antioxidantes do kaempferol-3-7-O-diramnosideo isolado da fração butanólica da Bauhinia forficata Link. XVII Simpósio de Plantas Medicinais do Brasil, Cuiabá, MT, Brazil, p. FT.185.

Sousa E, Cunha-Júnior A, Charão CCT, Zanatta L, Szpoganicz B, Pizzolatti MG, Jorge AP, Silva FRMB 2002b. Efeito agudo do extrato bruto de Vitex megapotamica (Verbenaceae) na glicemia de ratos normais, 
normais hiperglicêmicos e diabéticos induzidos com aloxana. VII Simpósio de Plantas Medicinais do Brasil, Cuiabá, MT, Brazil, p. FT.252.

Souza-Formigoni MLO, Lodder HM, Ferreira TMS, Carlini EA 1986. Pharmacology of lemongrass (Cymbopogon citratus Stapf). II. Effects of daily two month administration in male and female rats and in offspring exposed in utero. J Ethnopharmacol 17: 65-74.

Svoboda GH, Gorman M, Root MA 1964. Alkaloids of Vinca rosea (Catharanthus roseus). 28. A preliminary report on hypoglycemic activity. Lloydia 27: 361363.

Taylor TE, Molyneux ME, Wirima JJ, Fletcher KA, Morris K 1988. Blood-glucose levels in Malawian children before and during the administration of intravenous quinine for severe falciparum-malaria. $N$ Engl $J$ Med 319: 1040-1047.

Teixeira CC, Fuchs FD, Blotta RM, Knijnik J, Delgado IC, Netto MS, Ferreira E, Costa AP,. Mussnich DG, Ranquetat GG, Castaldo G 1990. Effect of tea prepared from leaves of Syzygium jambos on glucose tolerance in nondiabetic subjects. Diabetes Care 13: 907-908.

Teixeira CC, Pinto LP, Kessler FHP, Da-Paixão LQ,. Miura CS, Guimarães MS, Miura MS, Gastaldo GJ, Fuchs FD 1998. Is the decoction of mango leaves an antihyperglycemic tea?. Fitoterapia 69: 165-168.

Teixeira CC, Rava CA, Silva PM, Melchior R, Argenta R, Anselmi F, Almeida CRC, Fuchs FD 2000. Absence of antihyperglycemic effect of jambolan in experimental and clinical models. JEthnopharmacol 71:343-347.

Tomas GE, Lock O, Jurupe H 1999. Chemical study and hypoglycemic and hypolipemic activity of Gentianella thyrsoidea Hooker. Bol Soc Quim Peru 65: 231-238.

Tsiodras S, Shin RK, Christian M, Shaw LM, Sass DA 1999. Anticholinergic toxicity associated with lupine seeds as a home remedy for diabetes mellitus. Ann Emerg Med 33: 715-717.

Turner CE, Craig Jr JC 1974. Hypoglycemic compound. Patent US 3,922,263 4pp.

Vale DSD, MR. Kerntopf CK, Pontes MC, Fonteles M 2001. XVI Reunião Anual da Federação de Sociedades de Biologia Experimental, Caxambu, MG, Brazil, p. 319.

Valencia JM, Villavicencio R, Vicuña P, Torres E 1994. Efecto agudo del Aloe vera Linneo y Ciclanthera pedata Shrad en ratas diabéticas. Rev Med Peru 66: 80-82.

Volpato GT, Damasceno DC, Calderon IMP, Rudge MVC 1999. Study of Bauhinia forficata $\mathrm{L}$. extract on diabete in pregnant rats. Rev Bras Pl Med 2: 49-55.

Volpato GT, Damasceno DC, Calderon IMP, Rudge MVC 2002. Revisão de plantas brasileiras com comprovado efeito hipoglicemiante no controle do diabetes mellitus. Rev Bras Pl Med 4: 35-45.

Von Schmeling GA, Varela-de-Carvalho F, Domingos-Espinosa A 1977. Cienc Cult (Brazil) 29: 599-600.

Vuksan V, Stavro MP, Sievenpiper JL, Koo VY, Wong E, Beljan ZU, Francis T, Jenkins AL, Leiter LA,. Josse-Robert G, Xu Z 2000. American giseng improves glycemia in individuals with normal glucose tolerance: Effect of dose and time escalation. J Am College Nutr 19:

\section{8-744.}

Watters K, Blaisdell P 1989. Reduction of glycemic and lipid levels in $\mathrm{db} / \mathrm{db}$ diabetic mice by Psyllium plant fiber. Diabetes 38: 1528-1533.

White Jr JR, Kramer J, Campbell RK, Bernstein R 1994. Oral use of a topical preparation containing an extract of Stevia rebaudiana and the chrysanthemum flower in the management of hyperglycemia. Diabetes Care 17: 940-941.

Williams RH, Martin FB, Henley ED, Swanson HE 1959. Inhibitors of insulin degradation. Metabolism 8: 99113.

Witherup KM, McLaughlin JL, Judd RL, Ziegler MH, Medon PJ, Keller WJ 1995. Identification of 3-hydroxy3-methylglutaric acid (HMG) as a hypoglycemic principle of Spanish moss (Tillandsia usneoides). $J$ Nat Prod 58: 1285-1290. 